Proceedings of the Institution of
Civil Engineers
Maritime Engineering 159
March 2006 Issue MAI
Pages $9-24$
Paper 14154
Received $14 / 03 / 2005$
Accepted $04 / 1$ I/2005
Keywords:
coastal engineering/hydraulics \&
hydrodynamics/mathematical
modelling

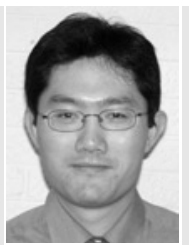

Ming Li

Lecturer, Department of Engineering, The University of Liverpool, UK

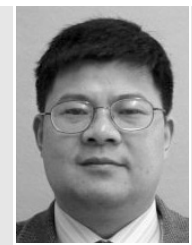

Shunqi Pan Lecturer, Department of Engineering, The University of Liverpool, UK

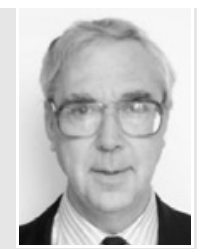

Brian A. O'Connor Emeritus Professor, Department of Engineering, The University of Liverpool, UK

\title{
Modelling coastal boundary layer flows over typical bed-forms
}

\section{Li MSc, PhD, S. Pan MSc, PhD and B. A. O'Connor Eur Ing, BEng, PhD, CEng, FICE}

This paper presents details of hydrodynamics, turbulence characteristics, sediment concentrations and transport rates computed by a new three-dimensional numerical boundary layer model above typical coastal bed-forms. Comparisons are made against a large number of available laboratory and field measurements covering situations of current alone, wave alone, and combined wave and current, which demonstrate the model's ability to simulate the complex structure of flow interactions as well as the associated sediment transport processes with reasonably good accuracy. The model results are also parameterised by averaging over both bed-form length and wave period in an attempt to produce improved relations of the overall bed-form-induced roughness height and equivalent near-bed reference concentration for engineering morphological modelling. Verifications of these averaged results against some simple engineering predictors show realistic agreements along with some noticeable differences. Where differences are found, modifications are also suggested to the existing engineering formulae in order to improve their predictions for complex flows. Further model testing is also suggested to refine the constants used for the proposed modifications.

\section{NOTATION}

$a$

$c$

$c_{\mathrm{a}}$

$c_{\mathrm{f}}$

$c_{\mathrm{p}}$

$D_{*}$

$d_{50}$

$d_{90}$

$f_{\mathrm{w}}$

$f_{\text {wc }}$

g

$h$

$k_{\mathrm{a}}$ wave orbital excursion diameter reference level for the near-bed sediment concentration calculation, $c_{a}$ suspended sediment concentration reference suspended sediment concentration at elevation $a$ above the mean bed level shear force drag coefficient pressure drag coefficient non-dimensional sediment particle size parameter sediment median particle size grain diameter for which $90 \%$ of the grain are finer by weight

friction factor for wave alone

friction factor for combined waves and current acceleration due to gravity

water depth

apparent roughness height in combined wave and current flows
$k_{\mathrm{S}}$

$k^{\prime}$

$k_{\mathrm{s}}^{\prime}$

$k_{\mathrm{s}}^{\prime \prime}$

$R^{2}$

$R e$

$R w$

$S$

$T$

$\boldsymbol{T}_{\mathrm{a}}$

$\boldsymbol{T}_{\text {current }}$

$T_{\mathrm{t}}$

$\boldsymbol{T}_{\text {wave }}$

$\boldsymbol{U}_{\mathrm{c}}$

$\boldsymbol{U}_{\infty}$

$\boldsymbol{u}$

$\boldsymbol{u}_{\mathrm{r}}$

$\boldsymbol{v}$

$\boldsymbol{w}$

$\boldsymbol{w}_{\mathrm{f}}$

$x$

$Y$

$\Delta$ $\varepsilon_{x}, \varepsilon_{y}, \varepsilon_{z}$ $\lambda$ $\lambda_{1}$ v total roughness height

scale factor in Grasmeijer et al. ${ }^{33}$ formula grain-related roughness height

bed-form-induced roughness height due to form drag

the coefficient of determination; namely the ratio of the sum of squares explained by a regression model and the total sum of squares around the mean

current-induced Reynolds number

wave-induced Reynolds number

sediment particle relative density

wave period

non-dimensional near-bed shear stress parameter wave-period-averaged current-related suspended sediment transport rate

wave-period-averaged total suspended sediment transport rate

wave-period-averaged wave related suspended transport rate

depth-averaged current velocity

near-bed wave orbital velocity magnitude

turbulence mean horizontal velocity in $x$ direction time-averaged and depth-averaged return velocity under a wave trough

turbulence mean horizontal velocity in $y$ direction turbulence mean vertical velocity in $z$ direction sediment particle fall velocity

longitude coordinate direction

vertical coordinate direction, measured positive upwards from the mean bed level

latitude coordinate direction

vertical coordinate direction, measured positive upwards from the bed

ripple roughness height scale factor

wave angle parameter

wave angle parameter

dune roughness scale factor for field conditions

ripple roughness scale factor to allow for ripples

sitting on dunes

bed-form height

sediment diffusion coefficients in $x, y$ and $z$

directions, respectively

bed-form wave length

length from bed-form crest to trough

fluid viscosity 
fluid density

sediment particle density

bed surface shear stress due to grain drag

bed shear stress due to form drag

critical shear stress for initial movement of sediment particles

the maximum shear stress throughout one wave cycle

angle between waves and current, in degrees

bed-form steepness parameter

wave angular frequency

wave-period-averaging operator

\section{INTRODUCTION}

Sediment transport is of major concern to coastal engineers due to the growing demand for prediction of shoreline and beach changes in the face of rising sea level. In recent years, computer modelling of coastal morphodynamics has become an increasingly popular way of tackling such a challenge as a result of the impressive development in computer technology. These engineering models often use a large-scale computational grid system with grid spacing up to hundreds of metres or kilometres in order to adequately cover a large coastal area. Unfortunately, such large grids are unable to directly represent the effects on sediment transport introduced by any small seabed features on the scales of centimetres or metres, for instance vortex shedding as surface waves propagate over a rippled bed (Fig. 1). At present, these small-scale bed features in the engineering models are usually represented by enhanced roughness elements with an effective height $\left(k_{\mathrm{s}}\right)$, while the sediment concentration is computed from a particular reference level $(a)$ above the grid-averaged bed level with a reference concentration $\left(c_{\mathrm{a}}\right)$. The accuracy of such a model, therefore, relies heavily on the accuracy of the prediction of the boundary conditions at the seabed. In addition, further engineering simplification may also be applied in engineering models by neglecting detailed variations within a wave period and adopting wave-period-averaged near-bed conditions instead. ${ }^{1}$ Although such models work well for current-dominated flows and coarse sediments, they are found to be less accurate for fine sediments due to significant intra-wave-period effects such as phase lag and wave-induced transport. Therefore, any improvement in the specification of near-bed processes would enable these large-area computer models to predict morphological changes more accurately. $^{2}$

Over the last decade, much research work has been carried out to study seabed boundary layer dynamics and the associated sediment transport processes through both small-scale laboratory experiments and field campaigns. However, most existing field measurements have been taken outside the boundary layer, and it has proved difficult to obtain accurate data near the bed-form surface because of the existence of highly turbulent flow conditions and the large near-bed sediment concentration gradients. Although experimental work in the laboratory has built some valuable datasets in the near-bed region for two-dimensional situations, for example Jensen et al., ${ }^{3}$ Ranasoma and Sleath, ${ }^{4}$ the physical scale of many tests often limits turbulence levels, which means that

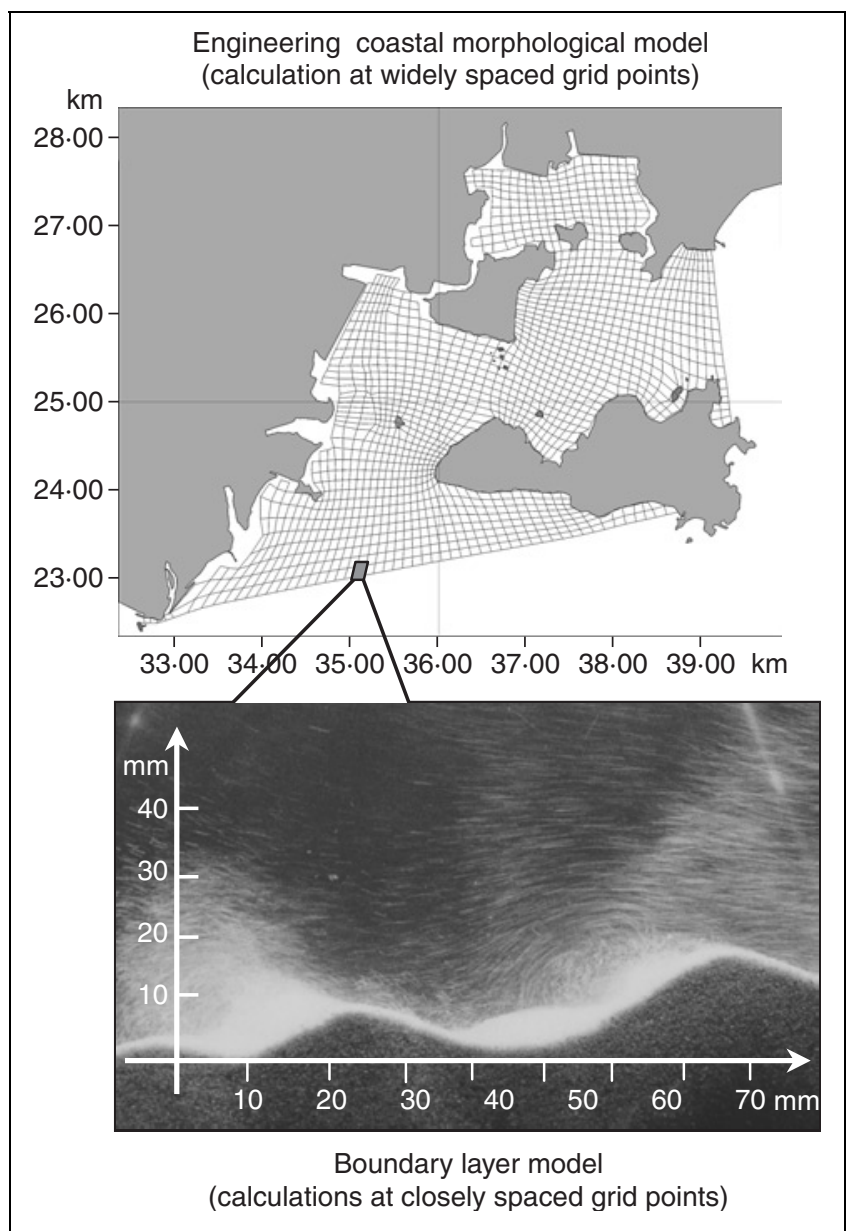

Fig. I. Schematic diagram for a large area engineering coastal model and a detailed bed-form-resolving boundary layer model

either very large facilities have to be employed in order to produce effects that are similar to the field conditions with absence of the effect of currents, or oscillating U-tubes are deployed to produce the conditions of waves and weak currents. Another problem which may have hindered the experimental studies in the past is the large quantity of data required to be obtained over a wide range of parameters, such as shear stress and turbulence levels, as well as the high quality of data which is difficult to obtain from the available electrical equipment with low sampling frequency. These problems have led researchers to develop boundary layer computer models to simulate near-bed flows and sediment transport rates (Fig. 1), see for example Hanson et al. ${ }^{5}$ and Andersen and Faraci. ${ }^{6}$ Unfortunately, the existing boundary layer models have been mostly used for two-dimensional cases, and there are as yet not many results for situations involving arbitrarily-angled wave and current flows. Furthermore, only limited attempts have been made to improve the accuracy of existing engineering predictors through parameterisation of results from these boundary layer research tools.

Li and O'Connor ${ }^{7}$ developed a general three-dimensional boundary layer model based on the CFD FLUENT ${ }^{8}$ package to study near-bed processes in the presence of arbitrarily-angled, combined wave and current flows over individual bed-forms. The model has been tested against a wide range of laboratory 
and field conditions. This paper focuses on analysing the results from these model tests and parameterising the results using a spatially (over the ripple length) and temporally (over the wave period) averaging method, so that simpler expressions of the effective bed roughness $\left(k_{s}\right)$, equivalent bed reference concentration $\left(c_{\mathrm{a}}\right)$ and the wave-related suspended sediment transport rate ( $\left.\boldsymbol{T}_{\text {wave }}\right)$ can be produced for use in large area engineering morphodynamic models, such as the 0'Connor et al. ${ }^{9}$ Q3D model.

\section{THE NUMERICAL MODEL}

The wave and current boundary layer model of Li and 0'Connor ${ }^{7}$ integrates the standard FLUENT ${ }^{8}$ package, which solves the three-dimensional Reynolds-averaged momentum and mass conservation equations for fluid flow, with additional new modules to simulate the wave motion and sediment transport required for the present study. Although full details of the model can be found elsewhere, it is worth mentioning the key features of the model here. Within the FLUENT package, a range of turbulence closure sub-models are available with different levels of complexity. In this study, a series of model tests have been carried out and the results indicated that it is necessary to use high-level closure in order to obtain better representation for the low Reynolds number flow found in the bed-form trough region. As a result, a Reynolds stress transport model was employed for turbulence simulation using the standard model coefficients without any special tuning for each individual case.

The standard FLUENT ${ }^{8}$ package can be used for complex flow predictions. However, it was found difficult to simulate the turbulence boundary layer flows under wave motions based on the existing modules within the package. Therefore, a new module was developed and integrated with FLUENT ${ }^{8}$ to simulate wave motions through an external oscillatory body force, similar to the work of Fredsøe et al. ${ }^{10}$ Wave and current interaction can also be included by adjusting the water surface slope after integration over one wave period.

Once the necessary hydrodynamic information was obtained, the instantaneous suspended sediment concentration was predicted by a new transport module integrated with FLUENT, ${ }^{8}$ using the mass conservation equation as follows

\begin{tabular}{|c|c|}
\hline & $\begin{aligned} \frac{\partial c}{\partial t} & +\boldsymbol{u} \frac{\partial c}{\partial x}+\boldsymbol{v} \frac{\partial c}{\partial y}+\left(\boldsymbol{w}-\boldsymbol{w}_{\mathrm{f}}\right) \frac{\partial c}{\partial z} \\
& =\frac{\partial}{\partial x}\left(\varepsilon_{x} \frac{\partial c}{\partial x}\right)+\frac{\partial}{\partial y}\left(\varepsilon_{y} \frac{\partial c}{\partial y}\right)+\frac{\partial}{\partial z}\left(\varepsilon_{z} \frac{\partial c}{\partial z}\right)\end{aligned}$ \\
\hline
\end{tabular}

where $c$ is volume concentration of suspended sediment; $t$ is time; $\boldsymbol{u}, \boldsymbol{v}$ and $\boldsymbol{w}$ are fluid velocities in the $x, y$ and $z$ directions; $\boldsymbol{w}_{\mathrm{f}}$ is the settling velocity of the sediment particle in the $z$ direction; $\varepsilon_{x}, \varepsilon_{y}$ and $\varepsilon_{z}$ are the sediment diffusivity coefficients in the $x, y$ and $z$ directions, respectively. These diffusivity coefficients were directly computed from the turbulence model. The coordinate system used in the model is shown in Fig. 2 for a two-dimensional bed-form geometry, where the $x$-axis is along the main bed-form length, perpendicular to the crest of the bed-form in the horizontal direction; the $y$-axis is placed parallel to the crest of the bed-form in the lateral direction; and the $z$-axis is the vertical coordinate starting from the seabed upwards towards the water surface.

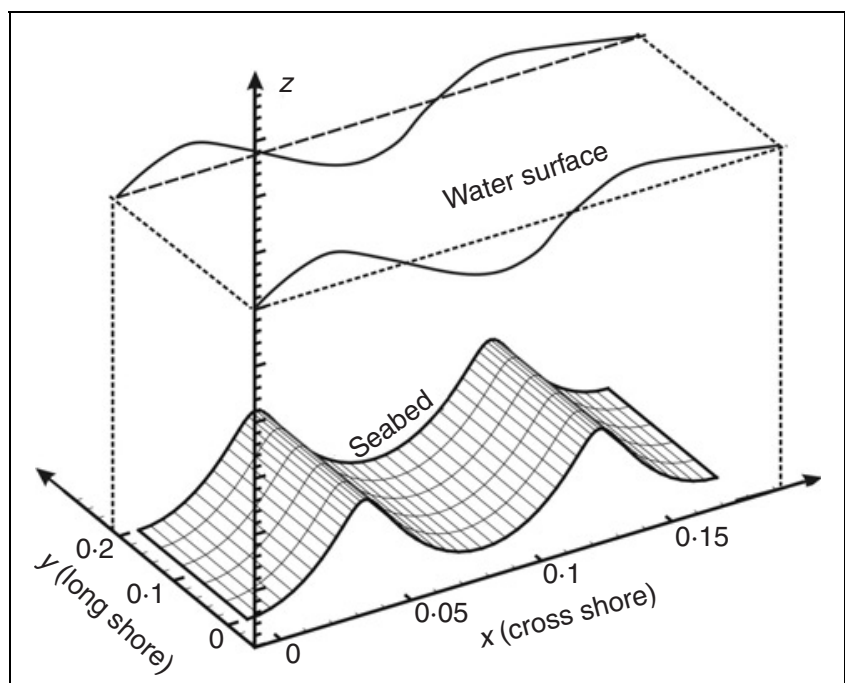

Fig. 2. Schematic diagram of the coordinate system used in the present numerical boundary layer model

To take sediment grading effects into account, the bed material was represented either by a single fraction with a representative grain size or as a range of fractions with different grain sizes.

In addition, a new curvilinear orthogonal grid generation module was also developed based on the approach of Eca ${ }^{11}$ in order to represent the curved surface of the bed features at the bottom boundary. Such a grid system allows grid space across the water column to vary so that sediment concentration distribution and transport at the seabed can be resolved in detail.

The model uses periodic conditions at two side boundaries and a slip boundary at the water surface. At the seabed, the flow velocities were taken to be zero. Sediment entrainment from the bed surface was represented by a gradient boundary condition similar to the approach of Fredsøe and Deigaard. ${ }^{12}$

The momentum and mass conservation equations for fluid flow were solved by the finite-volume method available in FLUENT on the curvilinear orthogonal grid as previously described. Equation (3) was solved on the same numerical grid by an implicit finite-difference approach to ensure the stability of the present numerical scheme.

\section{TEST CASES}

The model has been tested against a wide range of laboratory and field measurements available in the literature, totalling 32 cases (Tables 1-3). Among the 12 cases tested are those which involve unidirectional steady flows above large bed-forms, including the full-scale flow over artificial dunes in a large flume; ${ }^{13}$ laboratory measurements of flow above natural sandy ripples; ${ }^{14}$ laboratory experiments of flow over artificial bed-forms; ${ }^{15-18}$ and field measurements of tidal flow over large sandy dunes. ${ }^{19}$ Details of these test conditions are given in Table 1. Judging from the flow Reynolds number $(R e)$, it is apparent that all of these tests are within the turbulent flow region, although the laboratory tests are closer to the transitional region. Bed-forms in these tests include both small ripples of centimetre in height and metres in length in laboratory experiments and large sandy dunes up to several metres in the 


\begin{tabular}{|c|c|c|c|c|c|c|c|}
\hline Case & Author & $H: m$ & $U_{c}: \mathrm{m} / \mathrm{s}$ & $\operatorname{Re}\left(\times 10^{4}\right)$ & $d_{50}: m m$ & $\Delta / \lambda$ & Bed-form \\
\hline T5 & van Mierlo and de Ruiter ${ }^{13}$ & 0.254 & 0.450 & 11.40 & 1.6 & 0.050 & Dune \\
\hline T6 & van Mierlo and de Ruiter ${ }^{13}$ & 0.334 & 0.640 & 21.40 & 1.6 & 0.050 & Dune \\
\hline Raudkivi & Raudkivi ${ }^{14}$ & 0.145 & 0.300 & 4.35 & 0.2 & 0.078 & Ripple \\
\hline Case 2 & $\operatorname{Lyn}^{15}$ & 0.061 & 0.270 & 1.65 & - & 0.080 & Dune \\
\hline AR22 & Wiberg and Nelson'6 & 0.220 & 0.400 & $8 \cdot 80$ & - & 0.125 & Ripple \\
\hline$A R \mid 2$ & Wiberg and Nelson ${ }^{16}$ & 0.120 & 0.430 & $5 \cdot 16$ & - & 0.125 & Ripple \\
\hline Run I & $\mathrm{Li}^{17}$ & 0.100 & 0.130 & 1.30 & - & 0.100 & Ripple \\
\hline Run 2 & $\mathrm{Li}^{17}$ & 0.100 & 0.115 & 1.15 & - & 0.100 & Ripple \\
\hline Run 3 & $\mathrm{Li}^{17}$ & 0.100 & 0.117 & 1.17 & - & 0.100 & Ripple \\
\hline RI & Nelson and McLean ${ }^{18}$ & 0.195 & 0.510 & 9.95 & - & 0.050 & Dune \\
\hline R8 & Nelson and McLean ${ }^{18}$ & 0.220 & 0.410 & 9.02 & - & 0.125 & Ripple \\
\hline B3 & Atkins et al. ${ }^{19}$ & $3 \cdot 100$ & 0.650 & $201 \cdot 50$ & $0 \cdot 2$ & 0.053 & Dune \\
\hline
\end{tabular}

field measurements, and their steepness $(\Delta / \lambda)$ also varies from 0.05 to $0 \cdot 125$.

Table 2 summarises the 12 cases used in the model tests involving wave alone above vortex ripples, which include tests carried out for waves above a flat bed with enhanced roughness in a large U-tube; ${ }^{20}$ wave-induced flow above natural sandy ripples in U-tubes; ${ }^{21-23}$ waves above vortex ripples in both small and large wave flumes. ${ }^{10,24-27}$

The other eight cases for combined wave with current above rippled bed condition are listed in Table 3, including laboratory tests of waves with in-line current in a wave flume; ${ }^{10,27,28}$ and field measurements involving tidal current interacting with waves at a right angle in the surf zone. ${ }^{29}$ According to Jonsson, ${ }^{30}$ most of the cases in Tables 2 and 3 are within the rough turbulence region apart from one case of the Savell $^{24}$ tests. The steepness $(\Delta / \lambda)$ of the ripples ranges from $0 \cdot 07$ to around 0.2 , bed material covers fine $(0.09 \mathrm{~mm})$ to medium sand $(0.3 \mathrm{~mm})$, and the ratio between the wave orbital velocity magnitude $\left(\boldsymbol{U}_{\infty}\right)$ and depth-mean steady current velocity $\left(\boldsymbol{U}_{\mathrm{c}}\right)$ also varies from 1.0 to around 2.5 (see Tables 2 and 3).

\section{MODEL RESULTS}

Extensive numerical experiments have been firstly conducted with the present model to determine the optimal grid resolution and time step. Comparisons of the model results from different grid configurations suggested that the optimal grid resolution can be achieved when the vertical grid number was more than 60 and the horizontal grid size was less than $2 \%$ of the bed-form length or water depth, whichever was smaller. Therefore in general, model tests were carried out with 60 grid points over the flow depth and around 60-100 grid points over each bed-form length, which leads to horizontal and vertical grid sizes in the order of centimetres and millimetres, respectively. A typical time step was set at around 1/100 of the wave period to ensure the stability condition was satisfied; that is, the Courant number was less than 1. Further details of the numerical experiments can be found in $\mathrm{Li}^{31}$

The model was then applied to 32 cases as previously mentioned, to examine the detailed hydrodynamic and sediment transport processes within the boundary layer above the bed-form. The predicted flow velocities, turbulence characteristics and associated sediment concentration were compared with available measurements at a number of positions along the bed-form surface with the primary objective of evaluating the model's capability of reproducing transport processes under complex flows. It should be noted that only part of the model results from the selected tests are presented in this paper due to space limitations, and results not presented here were also found to be comparable to those shown. It also should be noted that all the cases presented herein involve two-dimensional wave-current conditions only. Details of three-dimensional simulations can be found in $\mathrm{Li}^{31}$

\begin{tabular}{|c|c|c|c|c|c|c|c|c|}
\hline Case & Author & $h: m$ & $T: S$ & $U_{\infty}: \mathrm{m} / \mathrm{s}$ & $A: m$ & $R w\left(\times 10^{4}\right)$ & $d_{50}: m m$ & $\Delta / \lambda$ \\
\hline No.I & Jonsson and Carlsen 20 & 0.30 & 8.4 & 2.11 & 2.85 & 601.4 & - & - \\
\hline Sleath & Sleath 21 & 0.19 & 4.5 & 0.11 & 0.08 & 0.9 & 0.20 & 0.17 \\
\hline Case 7 & Sato 22 & 0.21 & 4.0 & 0.30 & 0.19 & 5.6 & - & 0.17 \\
\hline T68, T69 & Savell $^{24}$ & 0.28 & 1.5 & 0.18 & 0.04 & 0.8 & 0.20 & 0.14 \\
\hline WI & Fredsøe et al. ${ }^{10}$ & 0.42 & 2.5 & 0.23 & 0.09 & $2 \cdot 1$ & - & 0.16 \\
\hline$\|$ & Nakato $^{23}$ & 0.25 & 1.8 & 0.27 & 0.08 & $2 \cdot 0$ & 0.14 & 0.14 \\
\hline TMR & Williams et al. ${ }^{25}$ & 4.50 & $5 \cdot 0$ & 0.70 & 0.56 & 38.9 & 0.31 & 0.17 \\
\hline T226 & Steetze| ${ }^{26}$ & 0.60 & 1.0 & 0.20 & 0.03 & 0.6 & 0.22 & 0.11 \\
\hline T235 & Steetzel $\left.\right|^{26}$ & 0.60 & 1.0 & 0.30 & 0.05 & 1.4 & 0.22 & 0.15 \\
\hline $\mathrm{T} 260$ & Steetzel ${ }^{26}$ & 0.60 & 1.0 & 0.50 & 0.08 & 4.0 & 0.22 & 0.13 \\
\hline $\mathrm{T} 264$ & Steetzel $^{26}$ & 0.60 & 1.0 & 0.75 & 0.12 & 8.9 & 0.22 & 0.07 \\
\hline T35 & Villaret and Perier ${ }^{27}$ & $1 \cdot 10$ & 1.5 & $0 \cdot 30$ & 0.07 & $2 \cdot 2$ & 0.09 & 0.15 \\
\hline
\end{tabular}




\begin{tabular}{|c|c|c|c|c|c|c|c|c|c|}
\hline Case & Author & $H: m$ & $T: s$ & $U_{\infty}: \mathrm{m} / \mathrm{s}$ & $A: m$ & $R w\left(\times 10^{4}\right)$ & $U_{\infty} / U_{c}$ & $d_{50}: m m$ & $\Delta / \lambda$ \\
\hline WCI & Fredsøe et al. ${ }^{10}$ & 0.42 & $2 \cdot 5$ & 0.23 & 0.09 & $2 \cdot 1$ & 1.00 & - & 0.16 \\
\hline T36 & Villaret and Perrier ${ }^{27}$ & 1.10 & 1.5 & 0.24 & 0.06 & 1.4 & 1.40 & 0.09 & 0.12 \\
\hline T38 & Villaret and Perrier ${ }^{27}$ & 1.10 & 1.5 & 0.23 & 0.05 & 1.2 & -1.30 & 0.09 & 0.13 \\
\hline$T 7 \cdot 5,10$ & van der Kaaij and Nieuwaar ${ }^{28}$ & 0.51 & $2 \cdot 6$ & 0.15 & 0.06 & 0.9 & 1.33 & 0.21 & 0.17 \\
\hline $\mathrm{T} 10,10$ & van der Kaaij and Nieuwaar ${ }^{28}$ & 0.52 & $2 \cdot 6$ & 0.21 & 0.07 & 1.4 & 1.78 & 0.21 & 0.19 \\
\hline $\mathrm{T} 12,10$ & van der Kaaij and Nieuwaar ${ }^{28}$ & 0.52 & 2.5 & 0.23 & 0.09 & $2 \cdot 1$ & 2.47 & 0.21 & 0.16 \\
\hline $\mathrm{T} I 5,10$ & van der Kaaij and Nieuwaar 28 & 0.50 & $2 \cdot 4$ & 0.28 & 0.11 & 3.0 & 2.62 & 0.21 & 0.18 \\
\hline Run I* & Saulter et al. 29 & 0.62 & 9.2 & 0.31 & 0.45 & 13.8 & $2.48 *$ & 0.30 & 0.17 \\
\hline
\end{tabular}

Table 3. Experimental conditions for combined waves with current tests: ( ${ }^{*}$ current at $90^{\circ}$ relative to wave propagation direction) $h$ is water depth, $T$ is wave period, $U_{\infty}$ is near bed orbital velocity, $A$ is the near-bed orbital excursion diameter, $U_{c}$ is the depth-mean velocity, $R w$ is the wave-induced Reynolds number $\left(U_{\infty} A / v\right), d_{50}$ is mean particle size, $\Delta$ is bed-form height, $\lambda$ is bed-form length

Comparisons of steady unidirectional current along a large-scale dune surface are presented in Fig. 3, in which the predicted horizontal velocity, turbulent kinetic energy and shear stress profile are shown together with the

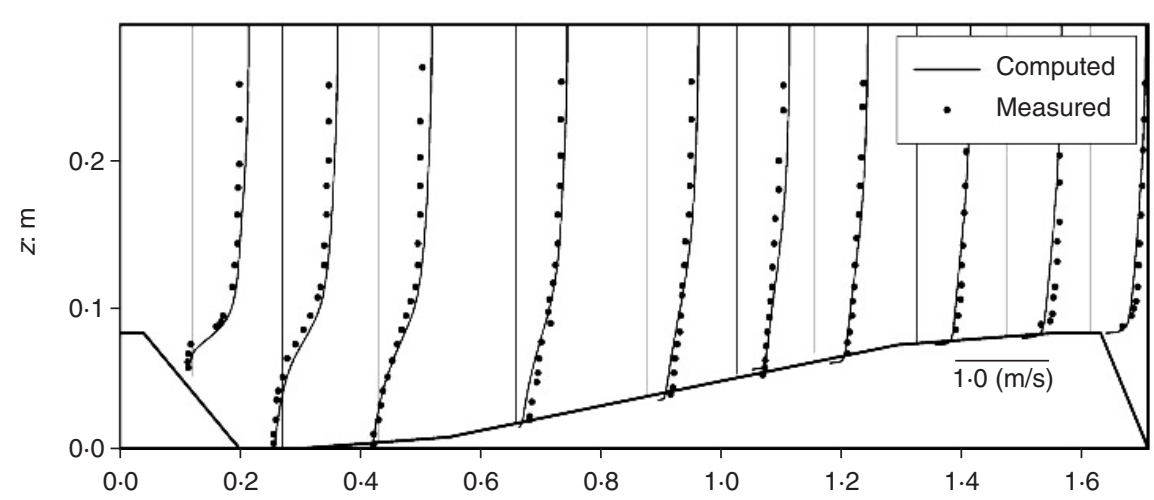

(a)

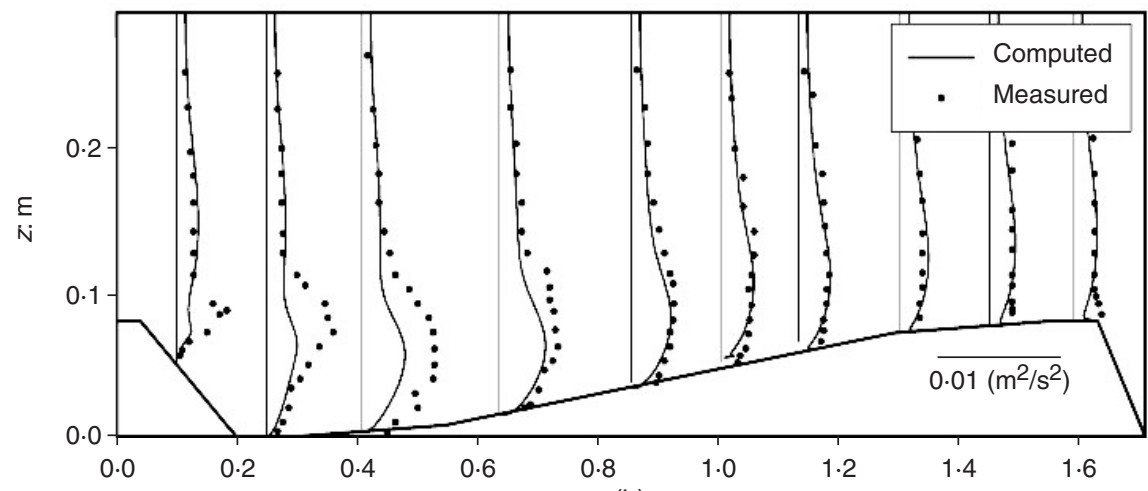

(b)

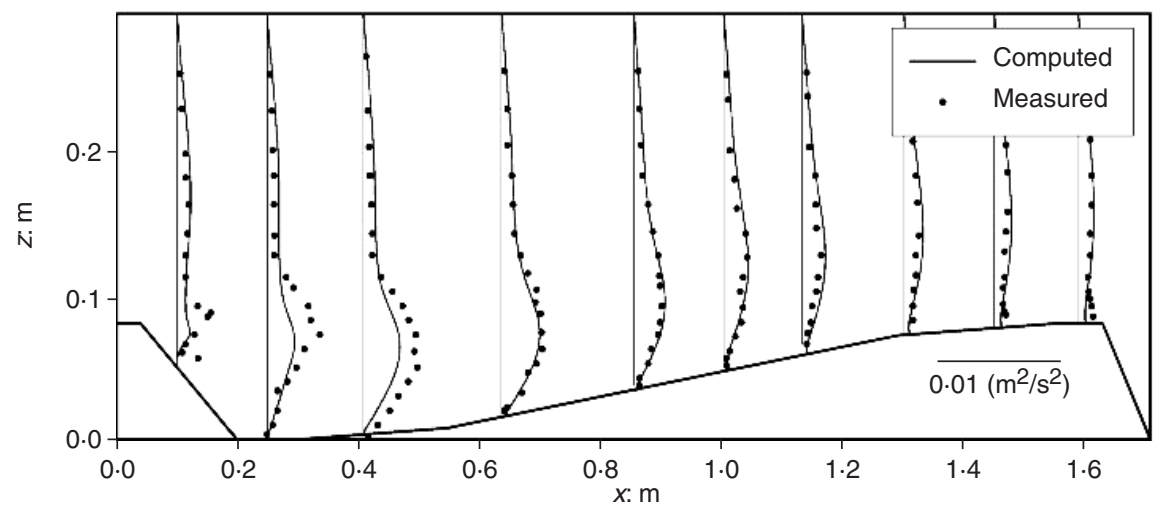

(c)

Fig. 3. Comparison of the computed vertical profiles of horizontal velocity (a), turbulent kinetic energy (b) and shear stress $\tau / \rho$ (c) at various locations along a bed-form with measurements of van Mierlo and de Ruiter ${ }^{13}$ (Test 6 - steady current) van Mierlo and de Ruiter ${ }^{13}$ experimental data. Overall, the model was found to be able to satisfactorily reproduce the important flow separation and reattachment processes in the trough region, a feature commonly observed in the boundary layer under unidirectional flows. However, the results also show that the turbulent energy and shear stress were underestimated above the trough area compared with the measurements, which indicates the difficulty in low Reynolds number turbulence simulation. Nevertheless, the averaged numerical error was found to be reasonably small: around $7 \cdot 8 \%$ for current velocity scaled by the depth-mean velocity, 11.5\% for turbulent kinetic energy and 10\% for shear stress based on the maximum measured values, respectively, with an assumption of no experimental errors. It should be pointed out that there are many other approaches that can also be used for assessing the present model's numerical errors, such as the Brier Skill Score. $^{32}$

The flow separation and reattachment also introduces a 'form drag'-that is, a certain part of the total drag due to the uneven distribution of the pressure forces along the bed-form surface (Fig. 4), in which the computed near-bed local shear force drag coefficient $c_{\mathrm{f}}$ and pressure drag coefficient $c_{\mathrm{p}}$, defined by equations (2a) and $(2 b)$, are compared for the 


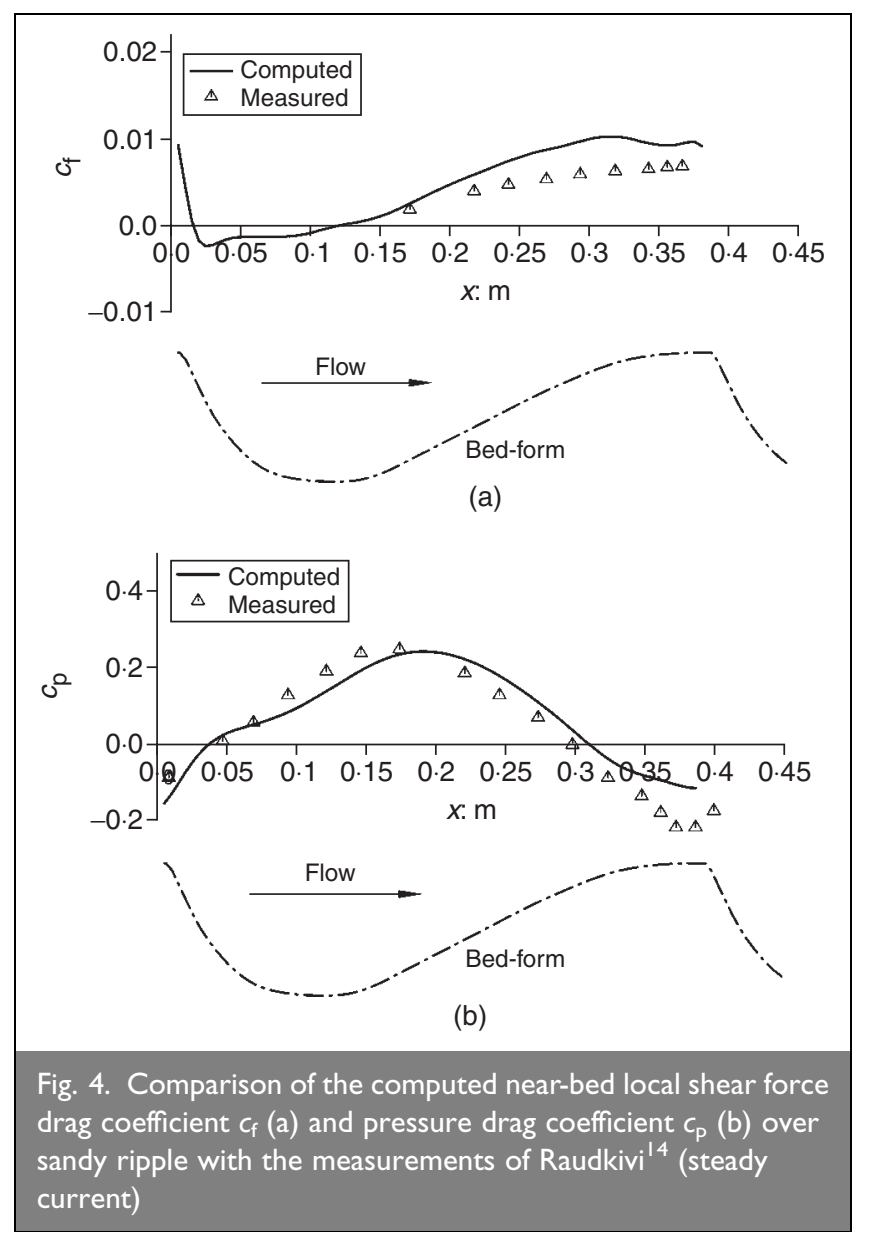

small-scale sandy ripple experiments of Raudkivi ${ }^{14}$

\begin{tabular}{|c|c|}
\hline $2 \mathrm{a}$ & $\mathrm{c}_{\mathrm{f}}=\frac{2 \boldsymbol{\tau}^{\prime}}{\rho \boldsymbol{U}_{\mathrm{c}}^{2}}$ \\
$2 \mathrm{~b}$ & $c_{\mathrm{p}}=\frac{2 \boldsymbol{\tau}^{\prime \prime}}{\rho \boldsymbol{U}_{\mathrm{c}}^{2}}$ \\
\hline
\end{tabular}

where $\boldsymbol{\tau}^{\prime}$ and $\boldsymbol{\tau}^{\prime \prime}$ are the skin friction and formal contribution to the total shear stress originated from the normal stresses (form drag), respectively; $\rho$ is the fluid density; and $\boldsymbol{U}_{\mathrm{c}}$ is the depth-mean velocity. The results clearly indicate that in the reversed flow zone, the shear stress changes to negative values due to the varying flow direction. In contrast, the computed and measured pressure coefficient reaches its maximum value in the trough region, and then decreases to a negative value before the crest.

For conditions involving waves above a rippled bed, a dominant feature in the boundary layer is vortex shedding within each half-wave cycle. The comparison between the model results and the Sato ${ }^{22}$ data in Fig. 5 shows the strong influence of such phenomenon on the flow distribution above ripples under asymmetrical waves, especially in the trough. For symmetrical waves, the model results tested against the Sleath ${ }^{21}$ experiments shown in Fig. 6 present a similar velocity distribution to a conventional oscillatory boundary layer flow over a flat bed with enhanced roughness. This is due to the fact that the wave orbital length in this case is small compared with the ripple length, and the vortex ejection tends to occur locally with evidently less influence on the flow structure higher in the water column. When a steady current is superimposed along with the wave oscillation, the flow field above the boundary layer or higher appears to be more controlled by currents. However, below the level of one to two ripple heights, the wave effects are found to be dominant. Such a feature can clearly be seen in Fig. 7, where the predicted horizontal velocity profiles at four sites along a vortex ripple are compared with the Fredsøe et al. ${ }^{10}$ experiment for waves superimposed with a following current at several wave phases. The overall agreements in Fig. 7 between the predictions and measurements are considered to be satisfactory, particularly higher in the water column.

However, differences can be found close to the ripple surface in the trough area at some wave phases. This is possibly due to the effects of surface wave asymmetry and the wave streaming induced by wave propagation, which is similar to the findings of Andersen and Faraci. ${ }^{6}$

For the sediment concentration distribution, both model predictions and the field measurements of Atkins et al. ${ }^{19}$ indicate the existence of a high concentration cloud within the bed-form trough area under a steady current, which leads to an unexpected reduction of concentration close to the bed surface, as shown in Fig. 8. However, concentration profiles at the crest and the other two positions along the bed-form show a normal decrease of concentration with increasing of the height in the water column. The underpredicted concentration at the crest in Fig. 8 also suggested that in the measurement certain sediment particles from the nearby dunes may be ejected over. In the oscillatory boundary layer, sediment movement is obviously affected by the vortex shedding and a significant amount of sand is rolled up along with the vortices during each half cycle. Fig. 9 presents the predicted concentrations at two levels above the crest and trough of a vortex ripple compared with the regular wave tests of Nakato. ${ }^{23}$ The distinct peaks during flow reversal are believed to be associated with vortex ejection events, which enhance sediment entrainment compared with a flat bed situation. In the presence of a steady current, the sediment transport tends to be pushed along in the current direction depending on the relative strength of the current and wave motion higher in the water column. However, in the area close to the ripple surface, the effects of the wave oscillation and the vortex shedding largely remain and have considerable impact. Such features can be seen evidently in Fig. 10, in which the predicted wave-period and ripple-length averaged total and wave-related suspended transport profiles are compared with the Villaret and Perrier ${ }^{27}$ measurements, namely

$$
\begin{array}{l|l}
\hline & \left\langle\boldsymbol{T}_{\mathrm{t}}\right\rangle=\boldsymbol{T}_{\text {current }}+\boldsymbol{T}_{\text {wave }}
\end{array}
$$

where $\boldsymbol{T}_{\mathrm{t}}$ is the total suspended sediment transport rate, $\boldsymbol{T}_{\text {current }}$ is the current related suspended transport rate, $\boldsymbol{T}_{\text {wave }}$ is the wave-related suspended transport rate and \langle\rangle denotes wave period averaging.

Table 4 lists the predicted suspended transport rates and the measurements from four cases in the van der Kaaij and Nieuwaar ${ }^{28}$ dataset and two tests of the Villaret and Perrier ${ }^{27}$ experiments (see Table 3). Overall agreements are considered to be satisfactory for the small number of tests, and the computed values are mostly within a factor of 2 of the measured values. The maximum divergence can be found in case T38, in which an opposing steady current was 

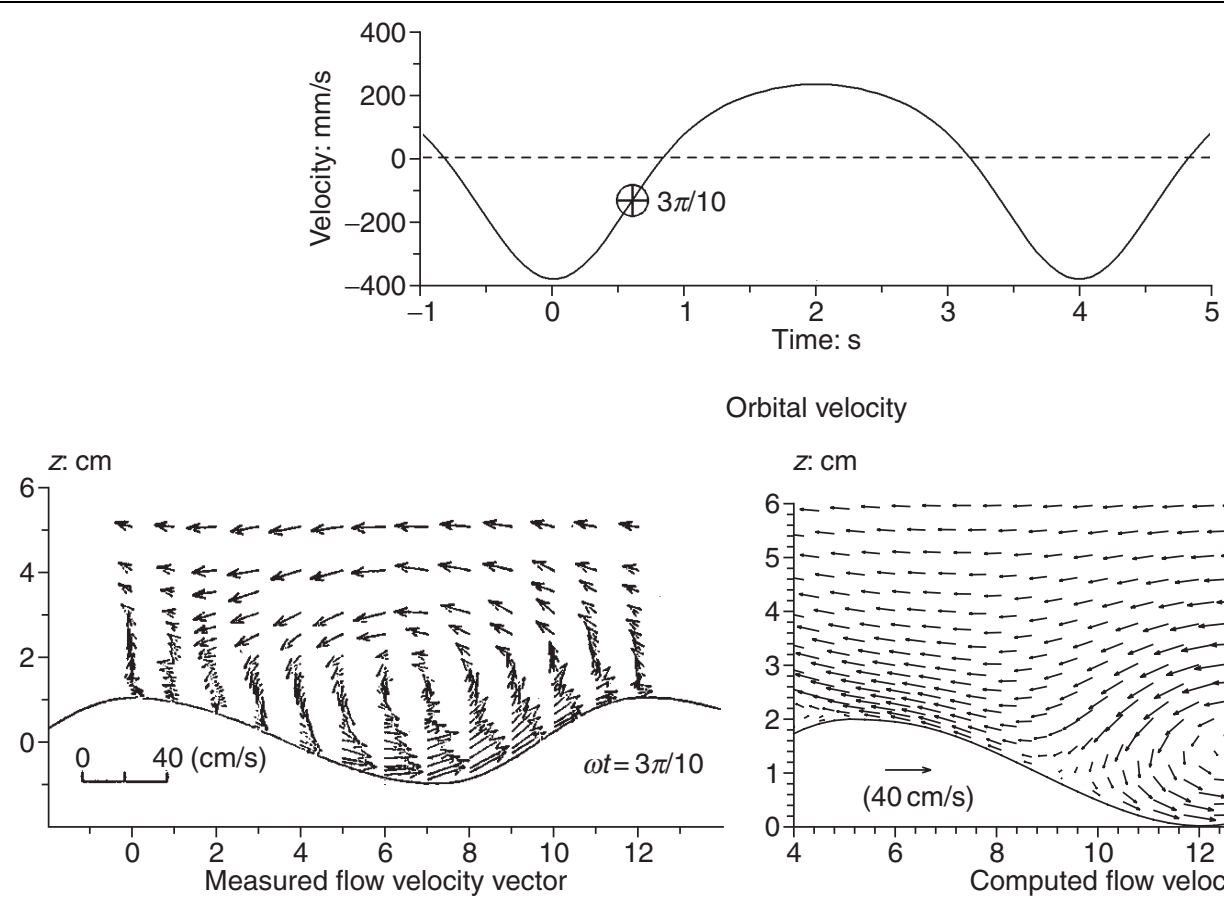

Orbital velocity

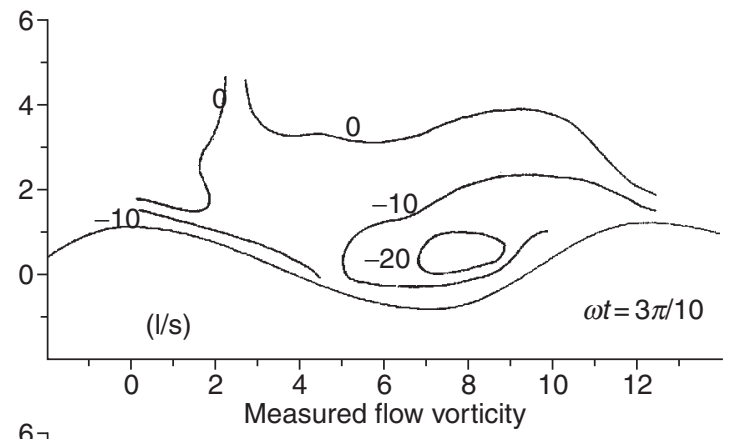

Z: $\mathrm{cm}$
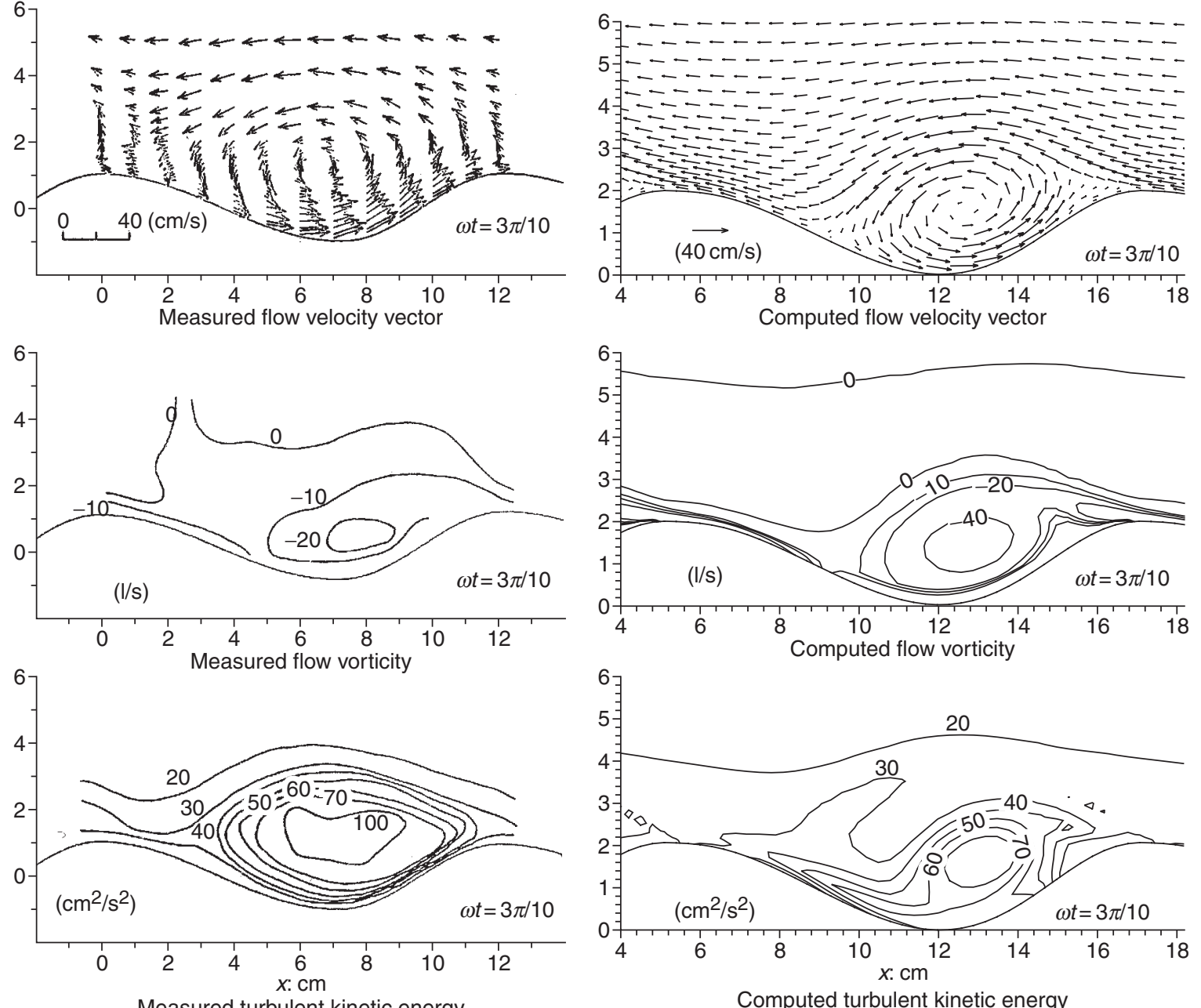

Fig. 5. Comparison of the computed flow velocity, vorticity and turbulent kinetic energy distribution above sandy ripple with the measurements of Sato ${ }^{22}$ (Case 7-asymmetrical wave)

superimposed above ripples formed by fine sand. Careful examination of the model results suggests that such difference is partly due to the overpredicted wave-related transport in the upper part of the water column along the current direction. It seems that in this experiment, the fine sand $(0.09 \mathrm{~mm})$ has introduced considerable phase lag which pushed more sediment opposite to the current direction than that predicted by the model. By comparison, the errors for the tests of van der Kaaij and Nieuwaar ${ }^{28}$ are much smaller where medium sand was used in the experiments. Clearly further study is needed for tests with fine sand. However, it should also be pointed out that no effect of measurement errors has been taken into account in the analysis due to the lack of information.
In addition to the above comparisons with the measurements, the computed wave-related suspended transport rates ( $\boldsymbol{T}_{\text {wave }}$ ) were also evaluated against a simple formula of Grasmeijer et $\mathrm{al}^{33}$ based on wave asymmetry and wave-period-averaged sediment concentration for cases T36 and T38 in Fig. 11. It is interesting to note that the present model results agree well with the predictions based on the approach of Grasmeijer et al. ${ }^{33}$ for case T36 with an error of 17\%, but the agreement is less satisfactory (79\%) for case T38. The scale-factor $\left(k^{\prime}\right)$ used for the approach of Grasmeijer et al. ${ }^{33}$ was kept as $0 \cdot 3$ as suggested. Due to the fact that the comparison was only made for a very limited number of tests, no general guideline can possibly be drawn at present as to the use of scale factor $\left(k^{\prime}\right)$ in their approach. 


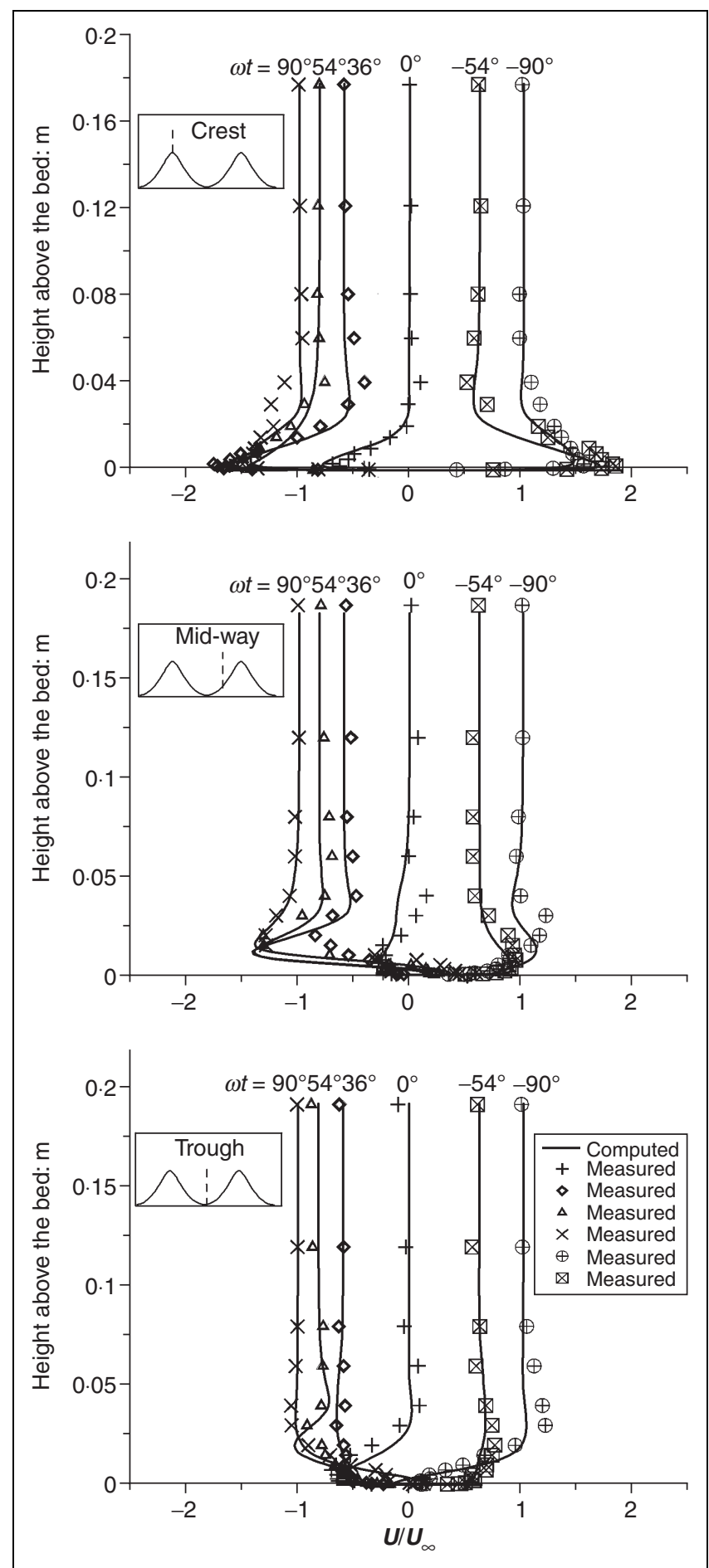

Fig. 6. Comparison of the computed vertical profiles of horizontal velocity at ripple crest, mid-way and trough with the measurements of Sleath ${ }^{21}$ at various wave phases (sinusoidal wave)

In order to examine the overall effect of bed-forms on sediment transport, the model results are parameterised by spatial (over the bed-form length) and temporal (over the wave period) averaging. The effective near-bed roughness $\left(k_{\mathrm{s}}\right)$ and equivalent reference concentration $\left(c_{\mathrm{a}}\right)$ obtained from such parameterisation are then compared with the various predictors currently widely used in engineering models.

Table 5 presents the computed effective roughness values for the bed-forms used in the present study from the computer model and the van Rijn ${ }^{1}$ approach detailed below, together with the laboratory and field measurements

$$
k_{\mathrm{s}}=k_{\mathrm{s}}^{\prime}+k_{\mathrm{s}}^{\prime \prime}
$$

where $k_{s}^{\prime}$ is the roughness height due to grains at the bed-form surface $\left(3 d_{90}\right)$ and $k_{\mathrm{s}}^{\prime \prime}$ is the roughness height due to form drag, that is

$$
\begin{array}{l|ll}
\hline 5 & k_{\mathrm{s}}^{\prime \prime}=1 \cdot 1 \gamma_{\mathrm{d}} \Delta\left(1-\mathrm{e}^{-25 \Delta / \lambda}\right) \text { for dunes }
\end{array}
$$

$$
6 \quad k_{\mathrm{s}}^{\prime \prime}=\alpha \gamma_{\mathrm{r}} \Delta\left(\frac{\Delta}{\lambda}\right) \text { for ripples }
$$

in which $\Delta$ is the bed-form height; $\lambda$ is the bed-form length; $\gamma_{\mathrm{r}}$ is a ripple presence factor allowing for the effects of ripples sitting on the back of a dune; and $\gamma_{\mathrm{d}}$ is a dune form factor allowing for field scale effects, both of which are taken as unity in the present study except the B3 case in Table $5\left(\gamma_{d}=0 \cdot 7\right)$. As suggested by other researchers, the $\alpha$ value can be taken from 16 to 25 and an average value of 20 was used in the present study. These test conditions included steady currents as well as combined waves and currents interacting above ripples, see Tables 1 and 3.

As can be seen from Table 5, the overall agreement of the model prediction is better with the measurements $\left(R^{2}=0.95\right)$ than the computed values using the approach of van $\operatorname{Rijn}^{1}\left(R^{2}=0.63\right)$. The van Rijn ${ }^{1}$ approach tends to underestimate ripple roughness compared with the present model results and the measurements in most situations. Such underprediction was also found in the previous work by Li and 0'Connor ${ }^{7}$ for steady currents using a range of bed-form sizes with the ripple steepness higher than $0 \cdot 1$. Similarly, Soulsby ${ }^{34}$ suggests a varying $\alpha$ value between 10 and 90 with a typical value of 30 . More recently, experimental work by Whitehouse et al. ${ }^{35}$ in a tidal flume also confirmed the above findings.

To clarify the differences between the present model and the approach of van Rijn, ${ }^{1}$ Fig. 12 presents the computed effective roughness heights from the present numerical model (crosses) together with the measurements listed in Table 5 (circles) and the corresponding values computed by equations (5) and (6) (straight line). Noticeable divergence can be found at the low and high ends of the bed-form steepness in the figure.

Based on the model results, a simple refinement of the approach of van Rijn ${ }^{1}$ can be suggested as equation (7) for ripples and equation (8) for dunes

\begin{tabular}{|l|l|}
\hline $7 \mathrm{a}$ & $k_{\mathrm{s}}^{\prime \prime} / \Delta=8 \Delta / \lambda$ for $0.025 \leqslant \Delta / \lambda<0.06$ \\
$7 \mathrm{~b}$ & $k_{\mathrm{s}}^{\prime \prime} / \Delta=35 \Delta / \lambda-1.62$ for $0.06 \leqslant \Delta / \lambda<0.2$ \\
$7 \mathrm{c}$ & $k_{\mathrm{s}}^{\prime \prime} / \Delta=22 \Delta / \lambda+0.98$ for $\Delta / \lambda \geqslant 0.2$ \\
\hline
\end{tabular}

for ripples and

\begin{tabular}{|l|l|}
\hline $8 \mathrm{a}$ & $k_{\mathrm{s}}^{\prime \prime} / \Delta=1.496-\chi \quad$ for $0.025 \leqslant \chi<0.23$ \\
$8 \mathrm{~b}$ & $k_{\mathrm{s}}^{\prime \prime} / \Delta=2.375-4.8 \chi$ for $0.23 \leqslant \chi<0.45$ \\
$8 \mathrm{c}$ & $k_{\mathrm{s}}^{\prime \prime} / \Delta=0.44-0.5 \chi$ for $0.45 \leqslant \chi<0.8$ \\
\hline
\end{tabular}

for dunes, where

9 $\chi=\exp [-25 \Delta / \lambda]$ 

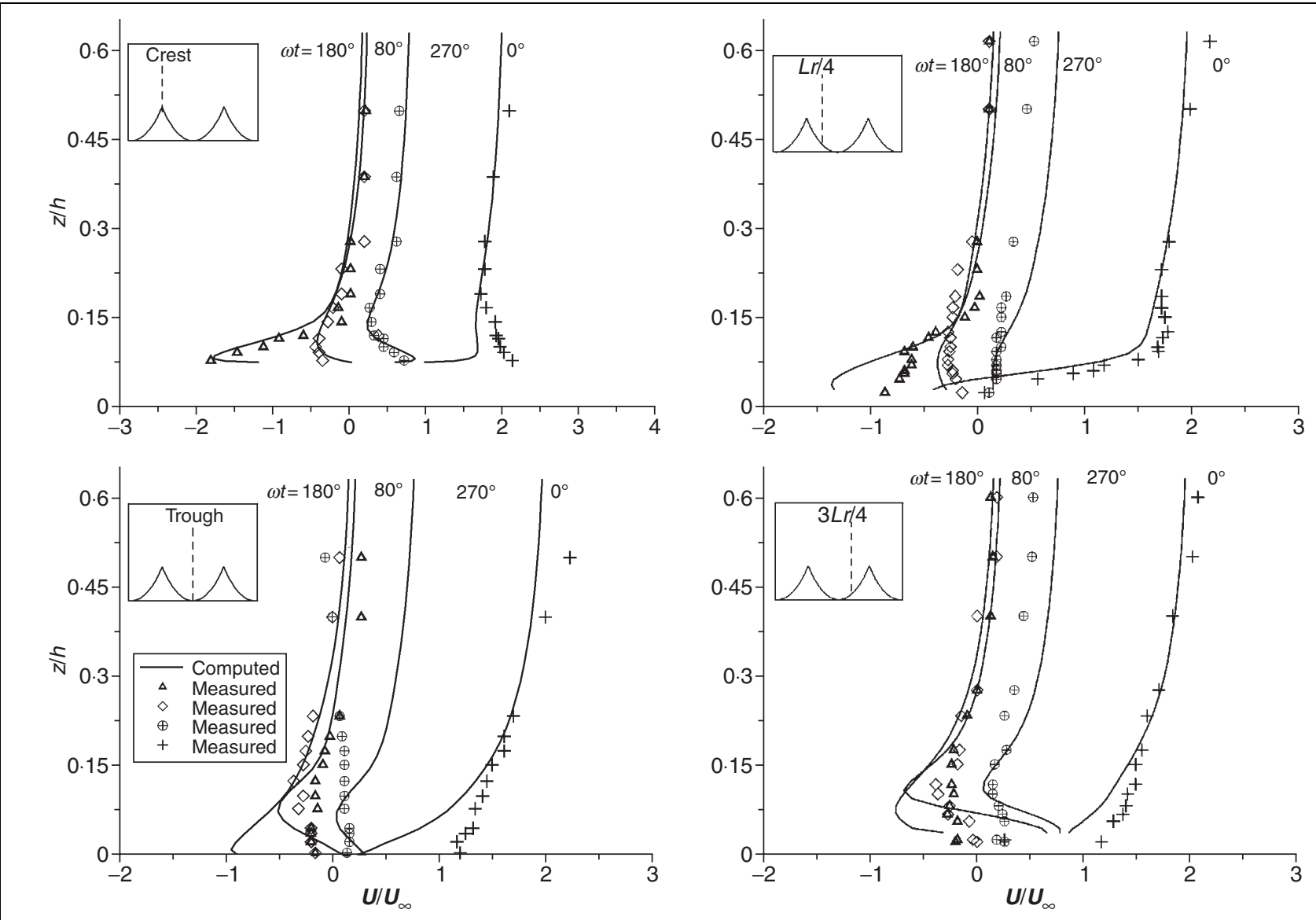

Fig. 7. Comparison of the predicted vertical profiles of horizontal velocity at four positions along a ripple with the measurements of Fredsøe et al. ${ }^{10}$ at various wave phases (WCI-wave with following current)

The results computed from equations (7) and (8) are also shown in Fig. 12 using broken lines and Table 5. It is evident that, on average, the new equations give an improved prediction of bed roughness in comparison with the method of van Rijn $^{1}$ with a better $R^{2}$ value (0.72). More importantly, the new formulae closely follow the same trend of the roughness height for the wide range of the bed-form steepness variation as the measurements. Only in few cases, however, the method of van Rijn ${ }^{1}$ provides a closer agreement with the measurements than those given by equations (7) and (8), which illustrates the difficulty in attempting to generate a set of universal equations for the full range of bed-forms. Therefore there is a need for more measurement data for the further refinement, as well as the possible necessity of introducing a more complex relationship for evaluating the effective roughness than the simple correlations of equation (5) and (6).

A further factor believed to have effect on the variability of bed roughness is the geometry of the bed-form. Further tests with the present model in $\mathrm{Li}^{31}$ show a significant reduction in form drag as the downstream slope of the bed-form $\left(\Delta / \lambda_{1}\right)$ is reduced, as shown in Fig. 13, compared with the results produced in the earlier study of Li and 0'Connor, ${ }^{7}$ which employed a standard bed-form shape suggested by the Raudkivi ${ }^{14}$ tests (see Fig. 4). For the bed-form with a sharp crest, the value of $\alpha$ in equation (6) therefore is expected to rise significantly, which may partly explain the three large enhanced roughness values found in the measurements of $\mathrm{Li}^{17}$ compared with equation (7) in Fig. 12(a). Based on a detailed intra-wave-period numerical model results, Kim et al. ${ }^{36}$ also found a very high $\alpha$ value (41) for sharp-crested ripples under waves.

For the combined wave and current situations, the apparent roughness height $k_{\mathrm{a}}$, due to wave and current interaction above a rough bed, is a very important parameter in boundary layer simulations. In the present study, the apparent roughness was obtained by fitting a logarithmic function through the wave-period-averaged residual flow profile above the bed-form crest. Van Rijn ${ }^{1}$ suggests that $k_{\mathrm{a}}$ can be related to the near-bed roughness value $k_{\mathrm{s}}$ by the following formulae

\begin{tabular}{ll}
\hline $10 \mathrm{a}$ & $k_{\mathrm{a}}=k_{\mathrm{s}} \exp \left[\frac{\gamma \boldsymbol{U}_{\infty}}{\sqrt{\left(\boldsymbol{U}_{\mathrm{c}}^{2}+\boldsymbol{u}_{\mathrm{r}}^{2}\right)}}\right]$ \\
$10 \mathrm{~b}$ & $\gamma=0 \cdot 8+\beta-0 \cdot 3 \beta^{2}$ \\
$10 \mathrm{c}$ & $\beta=\pi \phi / 180$ \\
\hline
\end{tabular}

where $\phi$ is the angle between the wave and current in degrees and $\boldsymbol{u}_{\mathrm{r}}$ is the depth-mean return flow velocity beneath the wave trough. For the test cases used in this paper, the current is either following or opposing the wave propagation direction and $\phi$ is therefore set at either zero or $180^{\circ}$. Another widely used approach to evaluate the wave-current interaction is the Fredsøe and Deigaard ${ }^{12}$ model in which the instantaneous flow velocity is computed, based on two prescribed logarithmic profiles for outside the boundary layer and inside the boundary layer, respectively, equivalent to a simplified one-dimensional intra-wave-period model, such as in 

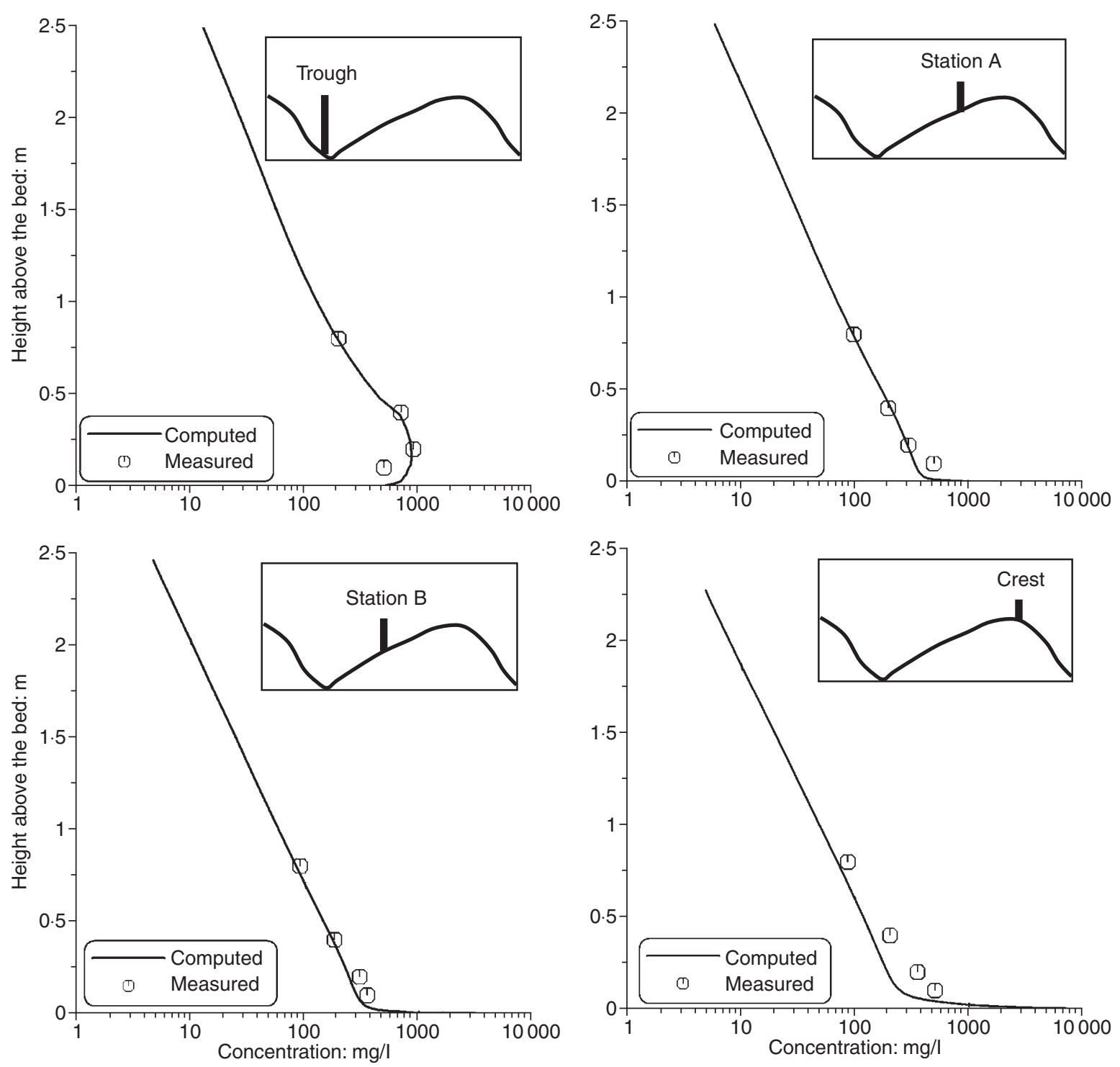

Fig. 8. Comparison of the computed vertical profiles of sediment concentration at various locations along a sandy dune with the field measurements of Atkins et al. ${ }^{19}$ (steady current)

Davies et al. ${ }^{37}$ Comparisons of the results obtained from the present model, equation (10) and the model of Fredsøe and Deigaard $^{12}$ are presented in Table 6. It is worth pointing out that in the method of Fredsøe and Deigaard, ${ }^{12}$ no specific formulae for the near-bed roughness calculation was suggested; instead, the measured values of $k_{\mathrm{s}}$ or those produced by the present model were used as data input in order to eliminate any additional uncertainties. Meanwhile, a representative regular wave was used to simulate the irregular waves in the experiments of van der Kaaij and Nieuwaar, ${ }^{28}$ which was also adopted in the present model, see Li. ${ }^{31}$ Overall, the computed resultant $k_{\mathrm{a}}$ values by the model are closer to those obtained from the method of Fredsøe and Deigaard ${ }^{12}$ than those of the approach of van Rijn, ${ }^{1}$ independent of wave and current relative strength (Table 6). However, in three cases, T10,10, T12,10 and $\mathrm{T} 15,10$, the computed roughness height by the present model agrees better with that predicted by the formula of van Rijn, ${ }^{1}$ which may suggest that better prediction can be achieved using equation (10) for relatively larger ripple steepness and wave/current ratios $\left(\boldsymbol{U}_{\infty} / \boldsymbol{U}_{\mathrm{c}}\right)$. These differences also indicate that with a proper use of near-bed roughness, the conventional one-dimensional engineering approach, such as the method of Fredsøe and Deigaard, ${ }^{12}$ is also likely to provide better flow profiles than the simple parameterised formula.

In the present work, model results for wave alone tests listed in Table 2 were also examined to determine the wave friction factor, $\boldsymbol{f}_{\mathrm{w}}$, which was then compared with the approaches of Swart, ${ }^{38}$ Antunes do Carmo et al. ${ }^{39}$ and Soulsby et $a l^{40}$ (Fig. 14(a)). In these friction factor evaluations, the effective ripple roughness height $k_{\mathrm{s}}$ from the present model were used instead of any empirical formula. Overall, the present model seems to follow the approaches of Antunes do Carmo et al..$^{39}$ and Swart ${ }^{38}$ reasonably well for all $A / k_{\mathrm{s}}$ values as shown in Fig. 14(a). It can be clearly seen that the results obtained from the approach of Soulsby et al. ${ }^{40}$ differ from those produced by other approaches for small $A / k_{\mathrm{s}}$ values, in particular, when $A / k_{\mathrm{s}}<3$.

For each combined wave-current case in Table 3, the computed maximum shear stress $\left(\tau_{\max }\right)$ was also compared with the approach of Soulsby and Clarke ${ }^{41}$ through the friction factor, $\boldsymbol{f}_{\mathrm{wc}}$, 


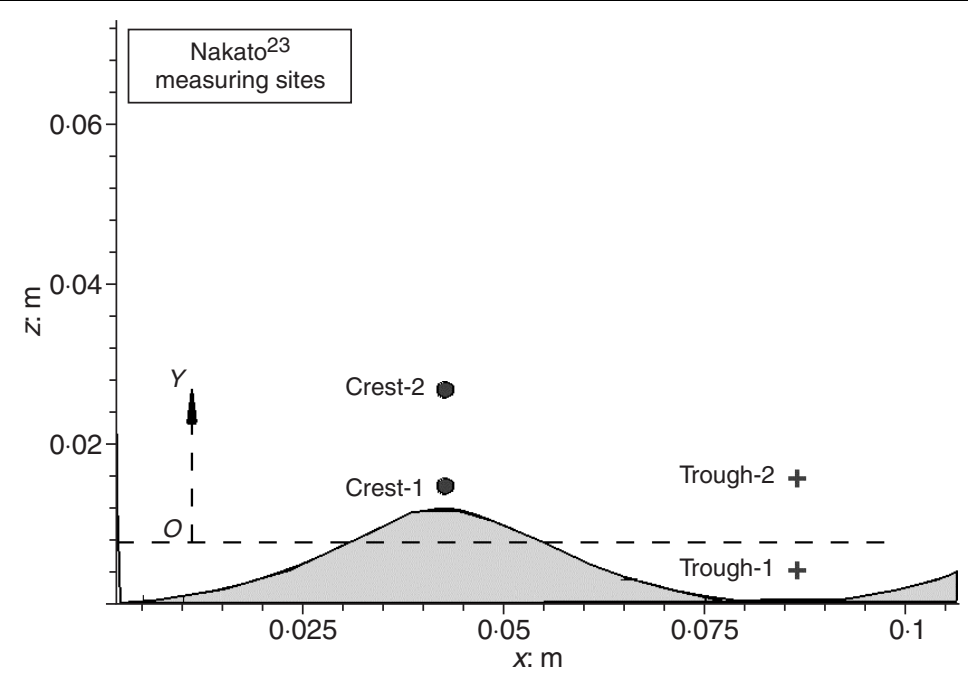

Crest
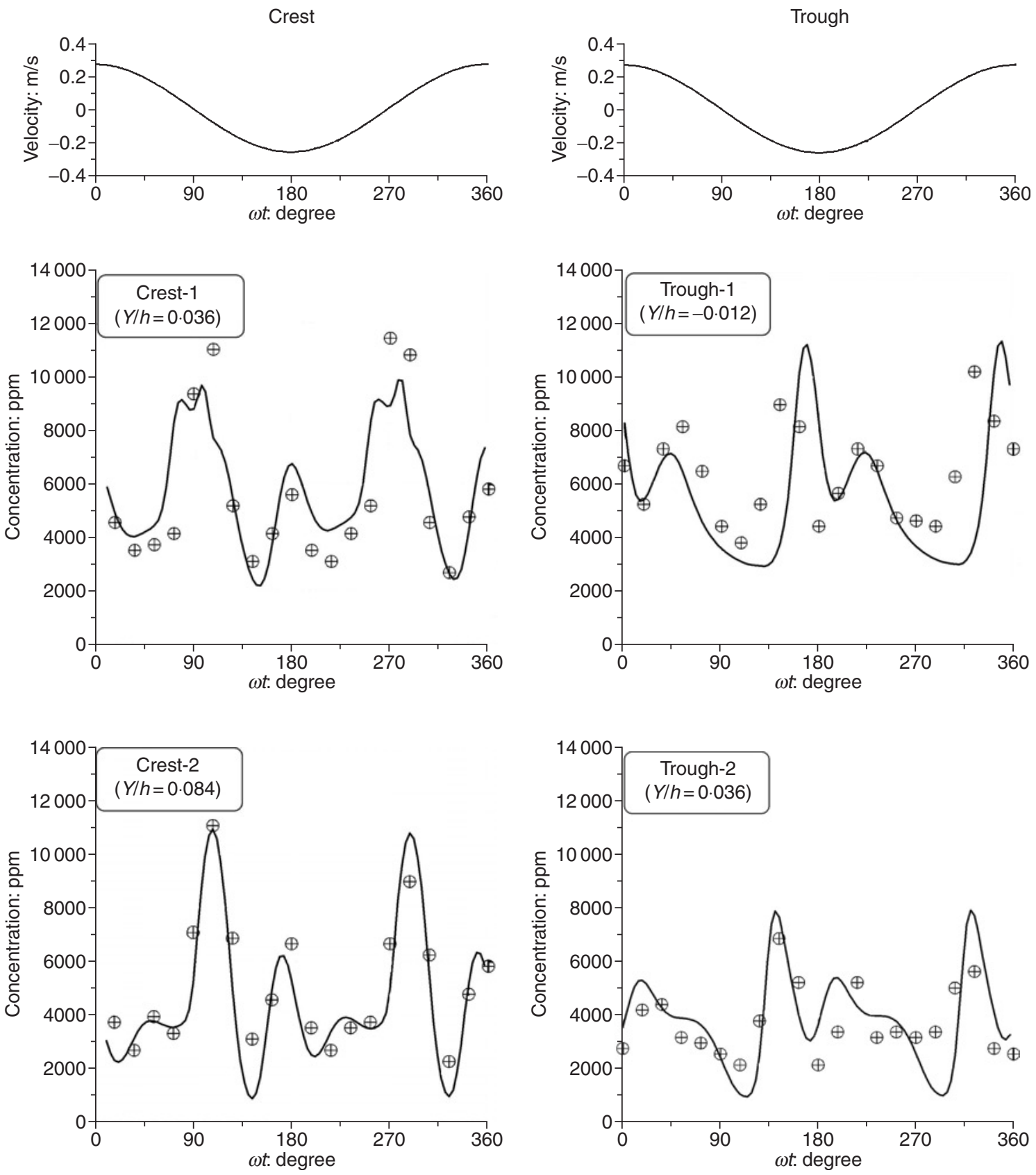

Fig. 9. Comparison of the computed time history of suspended sediment concentrations at two elevations above ripple crest and two elevations above ripple trough with the measurements of Nakato ${ }^{23}$ (sinusoidal wave case, the Yorigin is placed at the averaged depth between ripple crest and trough) 


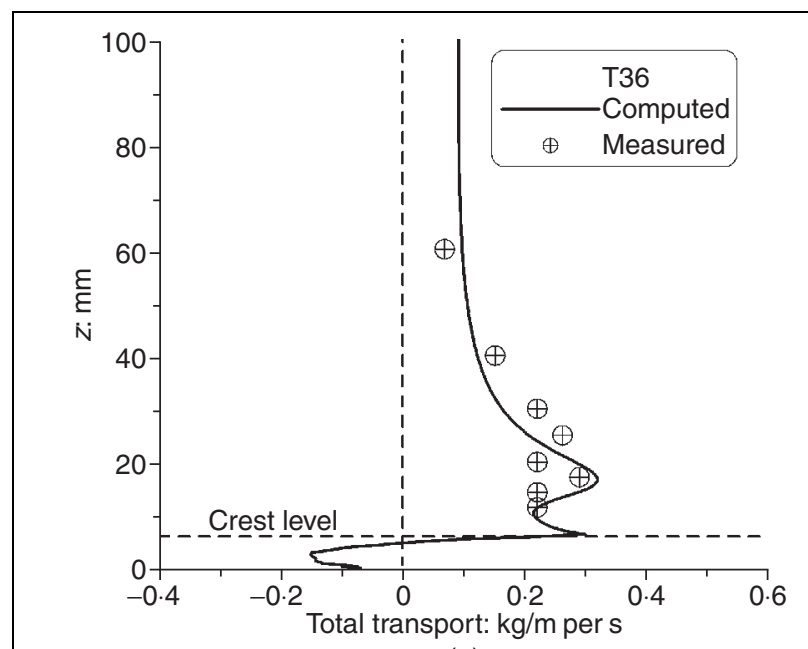

(a)

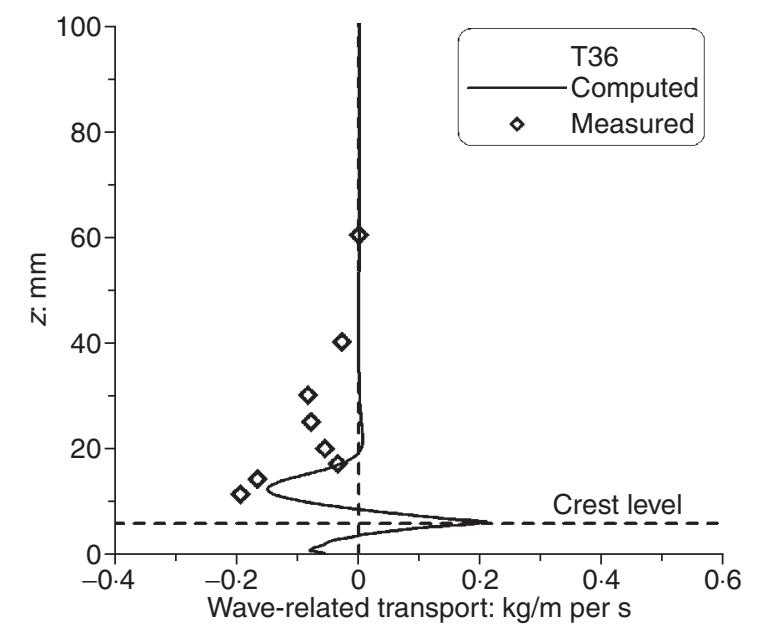

(b)

Fig. 10. Comparison of the computed profiles of wave-period and ripple-length averaged total suspended transport (a) and wave-related suspended transport (b) with the measurements of Villaret and Perrier ${ }^{27}$ (T36 - combined wave with following current)

defined based on the depth-mean current velocity as shown in Fig. 14(b).

\section{I}

$$
\boldsymbol{f}_{\mathrm{wc}}=\boldsymbol{\tau}_{\max } /\left[\rho \boldsymbol{U}_{\mathrm{c}}^{2}\right]
$$

It should be noted that the effective roughness height computed by the present model was employed in the estimations of $\tau_{\max }$ in the method of Soulsby and Clarke. ${ }^{41}$ The model results

\begin{tabular}{|lrcc|}
\hline & \multicolumn{3}{c|}{ Transport: $\mathrm{kg} / \mathrm{m}$ per $\mathrm{s} \times 10^{-3}$} \\
\cline { 2 - 4 } Case & Measured & Predicted & Error: $\%$ \\
\hline T7.5, 10 & 0.026 & 0.022 & 15 \\
T10,10 & 0.055 & 0.086 & 56 \\
T12,10 & 0.259 & 0.374 & 44 \\
T15,10 & 0.589 & 0.722 & 23 \\
T36 & 12.000 & 12.707 & 6 \\
T38 & 0.800 & -0.228 & 71 \\
\hline
\end{tabular}

Table 4. Comparison of the computed transport rates against measured values

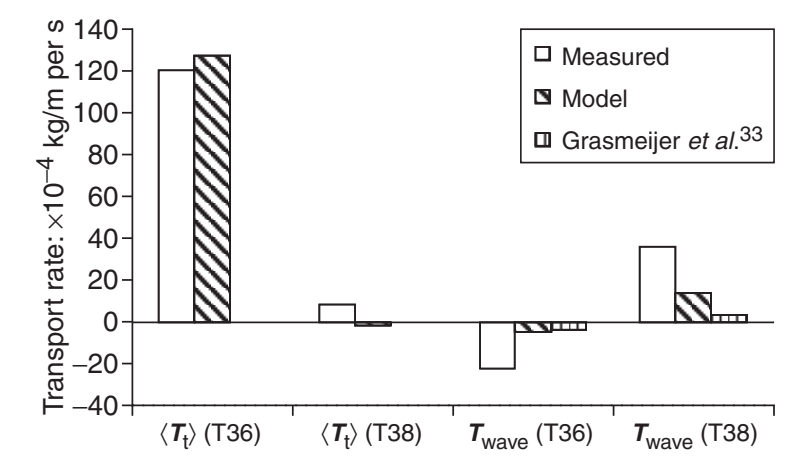

Fig. II. Comparison of the computed suspended

transport rates with the measurements of Villaret and Perrier ${ }^{27}$

(T36-wave with following current, T38-wave with opposing

current, $T_{\mathrm{t}}$-total transport rate and $T_{\text {wave }}$ - wave related

transport rate)

reasonably agree with those from the approach of Soulsby and Clarke $^{41}$ within an error of factor of 2, except for the two occasions of overprediction, which can be considered to be satisfactory in engineering applications. This agreement to a certain extent indicates the ability of the present model to predict the near-bed shear stresses under the combined wave and current conditions above rippled beds. The two overestimated points found in the T36 and T38 tests of Villaret and Perrier ${ }^{27}$ may be explained by the laboratory-scale effect due to the use of small laboratory facilities whereas the results of the approach of Soulsby and Clarke ${ }^{41}$ are primarily calibrated from field measurements.

In Fig. 15, the spatially-averaged model results from tests listed in Tables 1, 2 and 3 with mobile beds were also compared with the van Rijn ${ }^{1}$ predictions of near-bed reference concentration $\left(c_{\mathrm{a}}\right)$ calculated by the following formulae

\begin{tabular}{|l|l|}
\hline $12 \mathrm{a}$ & $c_{\mathrm{a}}=0.015 \frac{d_{50}}{a} \frac{\boldsymbol{T}_{\mathrm{a}}^{1.5}}{D_{*}^{0 \cdot 3}}$ \\
\hline $12 \mathrm{~b}$ & $\boldsymbol{T}_{\mathrm{a}}=\left(\boldsymbol{\tau}^{\prime}-\boldsymbol{\tau}_{\mathrm{cr}}\right) / \boldsymbol{\tau}_{\mathrm{cr}}$ \\
\hline $2 \mathrm{c}$ & $D_{*}=d_{50}\left[(s-1) \boldsymbol{g} / \boldsymbol{v}^{2}\right]^{1 / 3}$ \\
\hline
\end{tabular}

where $a$ is the reference height ( $1 / 2$ bed-form height); $\boldsymbol{\tau}^{\prime}$ is the grain related bed-shear stress; $\tau_{\mathrm{cr}}$ is the critical bed shear stress for initiation of sediment movement; $s=\rho_{\mathrm{s}} / \rho$ is the relative density of the sediment; $\boldsymbol{v}$ is the fluid viscosity and $\rho_{\mathrm{s}}$ is the density of the sediment. These tests include a steady current above a large sandy dune (Soulsby et $a l .{ }^{40}$ ), waves above vortex ripples (Steetzel ${ }^{26}$ ), and combined wave and current above vortex ripples (van der Kaaij and Nieuwaar ${ }^{28}$ ). It was found that for these tests equation (12a) provided a realistic prediction of $c_{a}$ (within $\pm 100 \%$ ). At the same time, it is also evident that under waves alone, equation (12a) seems likely to underpredict the near-bed reference concentration $c_{a}$, whereas for the combined wave and current conditions, it tends to overpredict the $c_{\mathrm{a}}$ value. To further improve equation (12a) for the combined waves and current situation, which is more desirable for engineering applications, a simple modification, as shown in Fig. 15, is also suggested as follows

$$
c_{\mathrm{a}}=\left[0.009 \frac{d_{50}}{a} \frac{\boldsymbol{T}_{\mathrm{a}}^{1.5}}{D_{*}^{0.3}}\right]^{1.43}
$$




\begin{tabular}{|c|c|c|c|c|c|c|c|}
\hline \multirow[b]{2}{*}{ Case } & \multirow[b]{2}{*}{ Bed-form } & \multirow[b]{2}{*}{ Condition } & \multirow[b]{2}{*}{$\Delta / \lambda$} & \multicolumn{4}{|c|}{$k_{s} / \Delta$} \\
\hline & & & & Measured & Model & van Rijn & Eqn $(7) /(8)$ \\
\hline T5 & Dune & C & 0.05 & 1.02 & 1.05 & 0.78 & 1.00 \\
\hline T6 & Dune & C & 0.05 & 0.93 & 0.76 & 0.78 & 1.00 \\
\hline B3 & Dune & C & 0.05 & - & 1.35 & $0.55^{*}$ & 1.00 \\
\hline Case 2 & Dune & C & 0.08 & 1.60 & 1.75 & 0.95 & 1.36 \\
\hline Raudkivi & Ripple & C & 0.08 & 0.47 & 0.42 & 1.60 & 1.18 \\
\hline RI & Ripple & C & 0.05 & 0.25 & 0.18 & 1.00 & 0.40 \\
\hline R8 & Ripple & C & 0.13 & 4.70 & 6.00 & 2.50 & 2.93 \\
\hline Run I & Ripple & C & 0.10 & 4.00 & $4 \cdot 20$ & 2.00 & 1.88 \\
\hline Run 2 & Ripple & C & 0.10 & 4.53 & 4.33 & 2.00 & 1.88 \\
\hline Run 3 & Ripple & C & 0.10 & 3.80 & 4.40 & 2.00 & 1.88 \\
\hline AR22 & Ripple & C & 0.13 & 4.75 & $6 \cdot 10$ & $2 \cdot 50$ & 2.93 \\
\hline WCI & Ripple & $W+C$ & 0.16 & 2.42 & 2.68 & 3.20 & 3.98 \\
\hline$T 7 \cdot 5,10$ & Ripple & $W+C$ & 0.17 & 4.06 & $5 \cdot 15$ & 3.40 & 4.33 \\
\hline TIO,IO & Ripple & $W+C$ & 0.19 & 4.88 & 4.85 & 3.80 & 5.03 \\
\hline $\mathrm{T} \mid 2,10$ & Ripple & $W+C$ & 0.16 & 4.88 & 6.23 & 3.20 & 3.98 \\
\hline TI5, I0 & Ripple & $W+C$ & 0.18 & 5.61 & $5 \cdot 50$ & 3.60 & 4.68 \\
\hline
\end{tabular}

Due to the fact that equation (13) was based on the results for combined wave and current cases, it can be seen that it is less suitable to those cases with wave or current alone that are shown in Fig. 15. However, such a simple modification reveals the possible improvement for equation (12a) that can be made using the present numerical model results given more comparison tests are available.

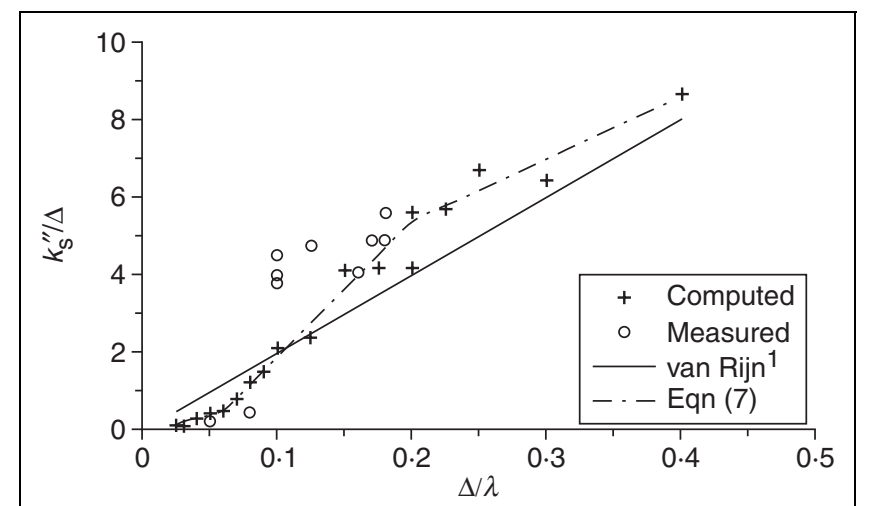

(a)

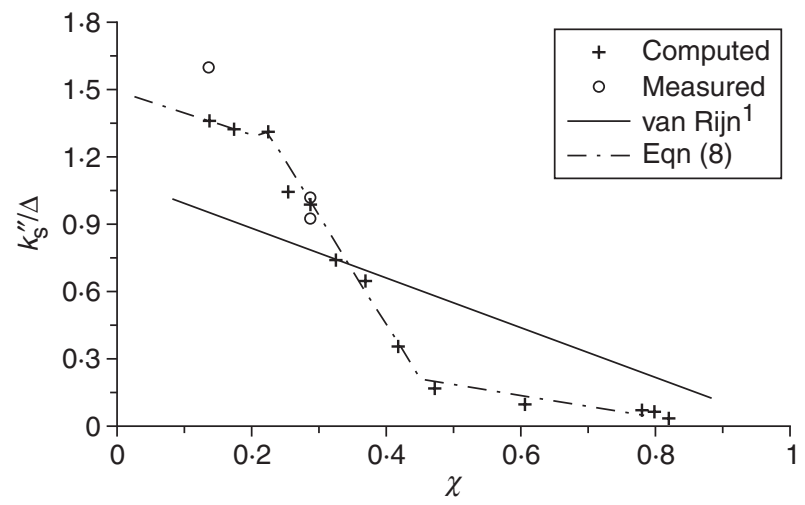

(b)

Fig. 12. Comparison of the computed bed-form effective roughness heights from the numerical model and the approach of van Rijn' with measurements for ripples (a) and dunes (b) with various bed-form steepnesses

\section{CONCLUSIONS}

Detailed model results obtained from a new three-dimensional numerical boundary layer model on hydrodynamics and sediment transport have been presented and analysed in this paper. The model tests included a wide range of current alone, wave alone and wave-current combined conditions on both the laboratory and field scales. Comparisons were made against the measurements, results calculated by commonly used formulae, as well as those from other numerical models, with satisfactory agreements.

By spatial and temporal averaging, the computed bed roughness, near-bed reference concentration, wave-induced and total suspended sediment transport have also been analysed and parameterised for engineering use with the aim of taking account of the effect of small-scale bed-form features and to provide better accuracy than the existing approaches. As a result, a new bed roughness formula has been suggested for various ripple and dune dimensions in equations (7) and (8). A new near-bed reference concentration prediction formula in

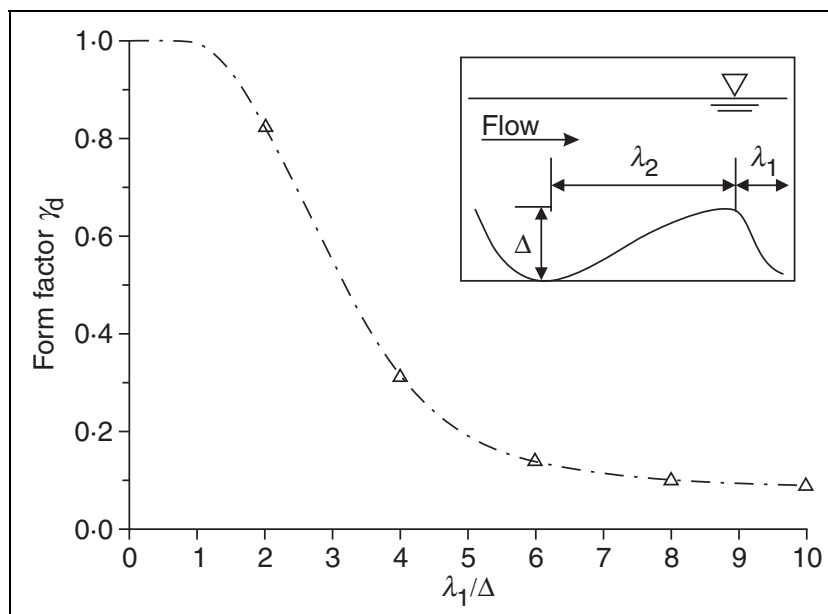

Fig. 13. Computed variation of form factor against bed-form steepness 


\begin{tabular}{|c|c|c|c|c|c|}
\hline \multirow[b]{2}{*}{ Case } & \multirow[b]{2}{*}{$\Delta / \lambda$} & \multirow[b]{2}{*}{$U_{\infty} / U_{c}$} & \multicolumn{3}{|c|}{$k_{\mathrm{a}} / \Delta$} \\
\hline & & & Model & van Rijn' & Fredsøe and Deigaard ${ }^{12}$ \\
\hline WCI & 0.16 & 1.00 & 24.4 & 7.1 & 21.9 \\
\hline T36 & 0.12 & 1.40 & 16.8 & 7.4 & 16.7 \\
\hline T38 & 0.13 & -1.30 & $20 \cdot 6$ & 6.9 & $28 \cdot 3$ \\
\hline$T 7.5,10$ & 0.17 & 1.33 & 23.4 & 9.9 & 20.0 \\
\hline TI0,10 & 0.19 & 1.78 & 19.1 & 15.8 & 27.1 \\
\hline $\mathrm{T} \mid 2,10$ & 0.16 & 2.47 & 21.8 & $23 \cdot 1$ & 18.8 \\
\hline TI5, I0 & 0.18 & 2.62 & 27.0 & 29.3 & $20 \cdot 1$ \\
\hline
\end{tabular}

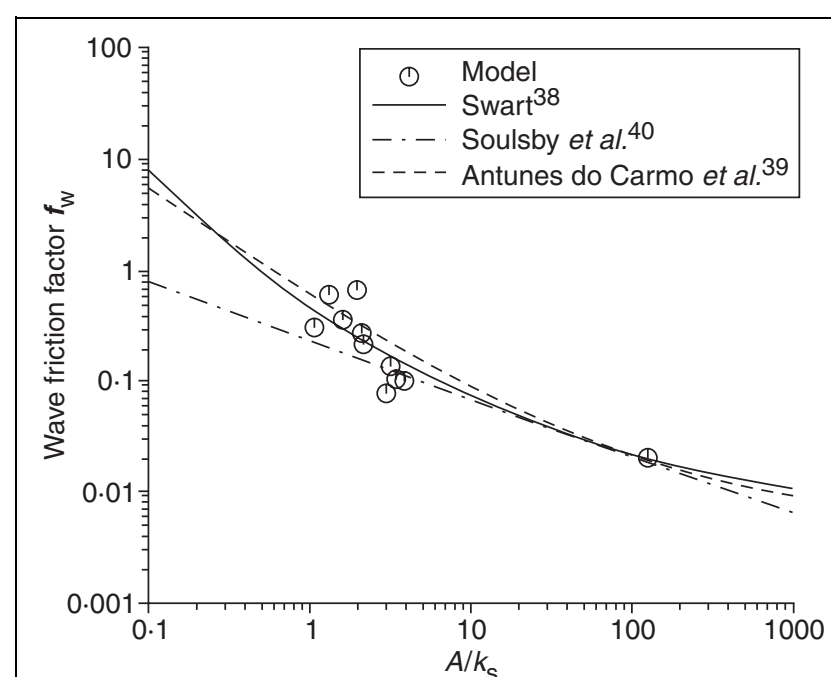

(a)

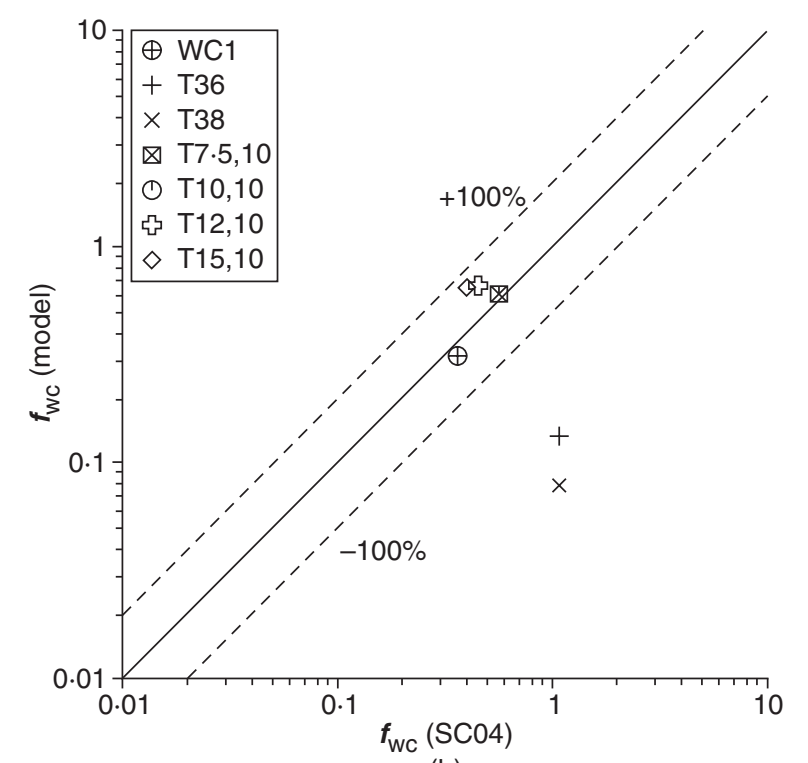

(b)

Fig. 14. Comparison of the computed wave friction factor $f_{w}$ and three empirical formulae in (a); and the computed combined-wave-current friction factor $f_{w c}$ with the method of Soulsby and Clarke ${ }^{41}$ (SC04) in (b)

equation (13) also has been proposed based on model results as an alternative to the commonly used approach of van Rijn. ${ }^{1}$ These two are important aspects in sediment transport predictions. Such improvements can now be readily implemented in engineering morphodynamic modelling systems. Clearly, for

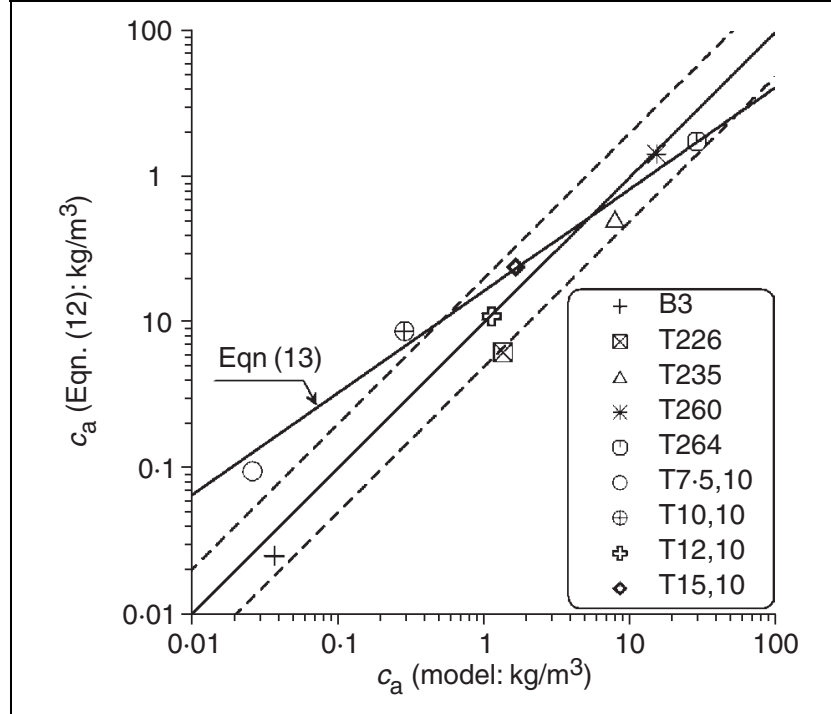

Fig. I5. Comparison of the computed reference concentration $\mathrm{C}_{\mathrm{a}}$ and equation $(\mathrm{I} 2 \mathrm{a})$

generic applications, the present model requires further calibration and parameterisation with more available laboratory and field data for various flow conditions and sediment characteristics. However, the model can be easily customised for a specific site to generate more detailed and accurate information on sediment transport for engineering use.

\section{ACKNOWLEDGEMENT}

This study was partly supported by the Engineering and Physical Sciences Research Council (UK) under COSMOD Project (Grant No: GR/R96967) and LEACOAST Project (Grant No: GR/R83545).

\section{REFERENCES}

1. VAN RIJN L. C. Principles of Sediment Transport in Rivers, Estuaries and Coastal Seas. Aqua Publications, Amsterdam, 1993.

2. O'Connor B. A. Suspended sediment transport in the coastal zone. Proceedings of an International Symposium on the Transport of Suspended Sediments and its Mathematical Modelling, Florence, Italy, 1991, pp. 17-63.

3. Jensen B. L., Sumer B. M. and Fredsøe J. Turbulent oscillatory boundary layers at high Reynolds numbers. Journal of Fluid Mechanics, 1989, 206, 265-297. 
4. Ranasoma K. and Sleath J. F. A. Velocity measurements close to rippled beds. Proceedings of the 23rd International Conference on Coastal Engineering, Venice, Italy. American Society for Civil Engineers, New York, 1992, pp. 2383-2396.

5. Hanson E., Fredsøe J. and DeigaArd D. Distribution of suspended sediment over wave-generated ripples. Journal of Waterway, Port, Coastal and Ocean Engineering, 1994, 120, No. 1, 37-55.

6. Andersen K. H. and Faraci C. The wave plus current flow over vortex ripples at an arbitrary angle. Coastal Engineering, 2003, 47, No. 4, 431-441.

7. LI M. and O'ConNOR B. A. Effective roughness of bed forms. In Hydraulic Engineering Software (BLAIN W. R. and Brebbia C. A. (eds)). WIT Press, Southampton, 2000, pp. 143-152.

8. FLUENT-INc. FLUENT 5 User's Guide. FLUENT-Inc., Lebanon, NH, USA, 1998.

9. O’Connor B. A., Pan S., Heron M., Williams J. J., Voulgaris G. and Silva A. Hydrodynamic modelling of a dynamic inlet. Proceedings of the 27th International Conference on Coastal Engineering, Sydney, Australia. American Society for Civil Engineers, New York, 2000, pp. 3472-3481.

10. Fredsøe J., Andersen K. H. and Sumer B. M. Wave plus current over a ripple-covered bed. Coastal Engineering, 1999, 38, No. 4, 177-221.

11. ECA L. 2D orthogonal grid generation with boundary point distribution control. Journal of Computational Physics, 1996, 125, No. 2, 440-453.

12. Fredsøe J. and Deigaard R. Mechanics of Coastal Sediment Transport. World Scientific, Singapore, 1992.

13. VAn Mierlo M. C. and DE RUITER J. C. Turbulence Measurements Above Artificial Dunes. Delft Hydraulic Laboratory, Delft, The Netherlands, 1988, Report No. Rep. Q789.

14. RAUdKIVI A. J. Bed forms in alluvial channels. Journal of Fluid Mechanics, 1966, 26, No. 3, 507-514.

15. LYN D. A. Turbulence measurements in open-channel flows over artificial bed forms. Journal of Hydraulic Engineering, 1992, 119, No. 3, 306-326.

16. Wiberg P. and Nelson J. M. Unidirectional flow over asymmetric and symmetric ripples. Journal of Geophysical Research, 1992, 97, No. C8, 12745-12761.

17. LI M. Z. Direct skin friction measurements and stress partitioning over moveable sand ripples. Journal of Geophysical Research, 1994, 99, No. C1, 791-799.

18. Nielson J. M. and McLean S. R. Flow and turbulence fields over two-dimensional bed forms. Water Resources Research, 1993, 29, No. 12, 3935-3953.

19. AtKins R., Soulsby R. L., WATERs C. B. and Oliver N. Field Measurements of Sediment Suspension above Bed forms in a Sandy Estuary. Hydraulic Research Limited, Wallingford, UK, 1989, Report No. SR203.

20. Jonsson I. G. and CARLSEN N. A. Experimental and theoretical investigations in an oscillatory turbulent boundary layer. Journal of Hydraulic Research, 1976, 14, No. 1, 45-59.

21. Sleath J. F. A. Ripple geometry under severe wave condition. Proceedings of the 27th International Conference on Coastal Engineering, Sydney, Australia. American Society for Civil Engineers, New York, 2000, pp. 2686-2699.
22. SATo S. Oscillatory Boundary Layer Flow and Sand Movement over Ripples. PhD thesis, University of Tokyo, 1987.

23. NAKATо T. Wave-induced Sediment Entrainment from Rippled Beds. PhD thesis, The University of Iowa, 1974.

24. SAVell I. A. An Experimental Study of Near-bed Hydrodynamics under a Combination of Waves and Steady Current and the Effects of this on Sediment Transport. $\mathrm{PhD}$ thesis, Department of Engineering, The University of Manchester, 1986.

25. Williams J. J., Bell P. S., Coates L. E., Hardcastle P. J., Humphery J. D., Moores S. P., Thorne P. D. and TrouW K. Evaluation of the Performance of STABLE. Proudman Oceanographic Laboratory, Liverpool, 1998, Report No. 53.

26. Steetzel H. J. Sediment Suspension in an Oscillating Water Motion Close to the Sand bed (in Dutch). PhD thesis, Delft University of Technology, The Netherlands, 1984.

27. Villaret C. and Perrier G. Transport of Fine Sand by Combined Waves and Current: An Experimental Study. Direction Des Etudes et Recherches, Electricité de France, 1992, Report No. E4202R.

28. VAn Der KaAiJ T. and NieuwaAr M. W. C. Sediment Concentrations and Sediment Transport in Case of Irregular Non-breaking Waves with a Current. Delft University of Technology, The Netherlands, 1987.

29. Saulter A., Russell P. and Huntley D. The effect of bed forms on coastal sediment transport. Proceedings of the 27th International Conference on Coastal Engineering, Sydney, Australia. American Society for Civil Engineers, New York, 2000, pp. 2672-2685.

30. Jonsson I. G. A New Approach to Oscillatory Rough Turbulent Boundary Layers. Coastal and River Engineering Section (ISVA), Technical University of Denmark, Lyngby, 1978, Series Paper 17.

31. LI M. Micro-modelling of Suspended Sediment Transport over Bed Forms. PhD thesis, Department of Civil Engineering, The University of Liverpool, 2004.

32. van Rijn L. C., Walstra D. J. R., GrasmeiJer B. T., Sotherland J., PAn S. and Sierra J. P. The predictability of cross-shore bed evolution of sandy beaches at the time scale of storms and seasons using process-based profile models. Coastal Engineering, 2003, 47, No. 3, 295-327.

33. Grasmeijer B. T., Chung D. H. and van RiJn L. C. Depth-integrated sand transport in the surf zone. In Proceedings of the 4th International Symposium on Coastal Engineering and Science of Coastal Sediment Processes held in Hauppauge, New York, June 21-23, 1999 (KraUs N. C. and McDougal W. G. (eds)). American Society for Civil Engineers, Reston, VA, 1999, pp. 325-340.

34. Soulsby R. L. Dynamics of Marine Sands. A Manual for Practical Applications. HR Wallingford, Wallingford, UK, 1997, Report No. SR 466.

35. Whitehouse R., Mitchener H. and Soulsby R. L. Ripple characteristics and bed roughness under tidal flow. In Conference Proceedings of the 3rd Coastal Dynamics Conference, held in Plymouth, UK, June 1997

(Thornton E. B. (ed.)). American Society for Civil Engineers, Reston, VA, 1997, pp. 1043-1052.

36. Kim H., O'Connor B. A. and Kim T. H. Effective bed roughness of wave friction factor over rippled bed. Proceedings of the 4th International Conference on Hydro-Science and Engineering, Seoul, Korea, 2000, 1-9 (CD Rom). 
37. Davies A., Soulsby R. and King H. A numerical model of the combined wave and current bottom boundary layer. Journal of Geophysical Research, 1998, 93, No. C1, 491-508.

38. SWART H. Predictive equations regarding coastal transports. Proceedings of the 15th International Conference on Coastal Engineering, Honolulu, Hawaii. American Society for Civil Engineers, New York, 1976, pp. 1113-1132.

39. Antunes do Carmo J. S., Temperville A. and Seabra-Santos F. J. Bottom friction and time-dependent shear stress for wave-current interaction. Journal of Hydraulic Research, 2003, 41, No. 1, 27-37.

40. Soulsby R. L., Hamm L., Klopan G., Myrhaug D., Simons R. and Thomas G. Wave-current interaction within and outside the bottom boundary layer. Coastal Engineering, 1994, 21, No. 1, 41-69.

41. SoulSBY R. L. and Clarke S. Bed Shear-stress under Combined Waves and Currents on Smooth and Rough Beds. HR Wallingford, Wallingford, UK, 2004, Report No. TR 137.

\section{What do you think?}

To comment on this paper, please email up to 500 words to the editor at journals@ice.org.uk

Proceedings journals rely entirely on contributions sent in by civil engineers and related professionals, academics and students. Papers should be 2000-5000 words long, with adequate illustrations and references. Please visit www.thomastelford.com/journals for author guidelines and further details. 